\title{
Hepatocyte serine palmitoyl transferase 2 deficiency promotes liver C16:0- ceramide accumulation through sphingomyelin hydrolysis and leads to liver damage and dysfunction in mice
}

\author{
Justine Lallement ${ }^{1}$, llyès Raho ${ }^{1}$, Gregory Merlen², Dominique Rainteau ${ }^{3}$, Mikael Croyal ${ }^{4}$, \\ Melody Schiffano ${ }^{4}$, Nadim Kassis ${ }^{1}$, Isabelle Doignon², Maud Soty ${ }^{5}$, Michel Krempf ${ }^{4}$, Chloé \\ Amouyal ${ }^{1}$, Hervé Le Stunff ${ }^{6}$ Christophe Magnan $^{1^{*}}$, Thierry Tordjmann ${ }^{*}$ and Céline Cruciani- \\ Guglielmacci ${ }^{*}$
}

1. Université de Paris, BFA, UMR 8251, CNRS, France.

2. Université Paris-Saclay, Inserm U1193, Orsay, France.

3. Sorbonne Université, Inserm, Centre de Recherche Saint-Antoine, CRSA, AP-HP, Hôpital Saint Antoine, Biochemistry Department, Paris, France.

4. Plateforme de Spectrométrie de Masse du CRNH-O, UMR1280, Nantes, France.

5. Université Claude Bernard Lyon 1, Université de Lyon, INSERM UMR-S1213, Lyon, France.

6. Institut des Neurosciences Paris-Saclay, CNRS UMR 9197, Université Paris Saclay, France

* : These authors contributed equally to this work. 


\section{$\underline{\text { Acknowledgment }}$}

This project has received funding from the Innovative Medicines Initiative 2 Joint Undertaking under grant agreement No 115881 (RHAPSODY). This Joint Undertaking receives support from the European Union's Horizon 2020 research and innovation programme and EFPIA.

We acknowledge the technical platform Functional and Physiological Exploration platform (FPE) for body composition analysis (Université de Paris, BFA, UMR 8251 CNRS, Paris, France), the animal core facility "Buffon" of the University de Paris, Institut Jacques Monod, especially Laëtitia Pontoizeau, for animal husbandry and breeding.

We also acknowledge Dr. Fréderic Preitner and Anabela Rebelo Pimentel Da Costa from the Mouse Metabolic Facility in Center for Integrative genomics, University of Lausanne, Lausanne, Switzerland for measurement of energy content in feces ; Nicolas Sorhaindo from biochemical analyses facility in Inflammation research center, (Université de Paris, UMR 1149 Inserm, ERL CNRS 8252, Paris, France) for plasma biochemical analyses and Hermine Kakanakou and Sylvie Le Marchand from the genotyping and biochemical facility, Cordeliers research center, (Sorbonne Université, Paris, France) for mouse genotyping.

We also acknowledge Amandine Gautier-Stein from Université Claude Bernard Lyon 1, (Université de Lyon, INSERM UMR-S1213, Lyon, France) for her scientific advice and protocols. 


\section{Abstract}

Objectives: Ceramides have been shown as lipotoxic inducers, which can trigger apoptosis, inflammation and disturb numerous cell signalling pathways leading to metabolic disorders such as type 2 diabetes (T2D). In this study, we aimed to determine the role of de novo hepatic ceramide synthesis on energy and liver homeostasis in mice.

Methods: In order to investigate hepatic role of de novo ceramides synthesis, we generated mice lacking serine palmitoyltransferase 2 (Spt/c2) in hepatocytes using the cre-lox system. SPTLC2 allows condensation of serine and palmitoylCoA and is the rate limiting-enzyme necessary for ceramide de novo synthesis. Sptlc2 $2^{\Delta \mathrm{Hep}}$ and their littermate controls were fed with high fat diet (HFD) to induce metabolic disorders. Liver ceramides content and metabolic parameters as glucose tolerance, insulin sensitivity, and hepatic glucose production were assessed. As ceramides may have impact on bile acids (BA), we investigated BA pool composition, synthesis and transport. Finally, inflammation and apoptosis were measured in the liver using western blot analysis, pro-inflammatory cytokines expression level and immunohistochemistry.

Results: Despite lower expression of hepatic Spt/c2, we observed an increased concentration of hepatic ceramides, especially C16:0-ceramide. Hepatic deletion of Sptlc2 in mice was also associated with an increased neutral sphingomyelinase 2 (nSmase2) expression, and a decreased sphingomyelin content in the liver. We showed that Sptlc2 ${ }^{\Delta \mathrm{Hep}}$ mice are protected against body mass gain normally induced by HFD and displayed a decreased body fat mass. BA hydrophobicity was drastically decreased in Sptlc2 $2^{\Delta \mathrm{Hep}}$ mice, and was associated with a defect in lipid absorption. In addition, an important increase of tauro-murocholic acid T-MCA in BA pool composition of Spt/c2 $2^{\Delta \mathrm{Hep}}$ mice was associated with a downregulation of the nuclear bile acid receptor FXR target genes in ileum and liver. Sptlc2 deficiency also enhanced glucose tolerance and attenuated hepatic glucose production in an insulin-independent manner. Finally, Sptlc2 disruption promoted progressive development of hepatic fibrosis, apoptosis and inflammation in an age-related manner.

Conclusion: Our data demonstrate for the first time a potential compensatory mechanism to regulate hepatic ceramides content from sphingomyelin hydrolysis. In addition, our results highlight the role of hepatic sphingolipid modulation on hepatic glucose production through bile acid composition changes.

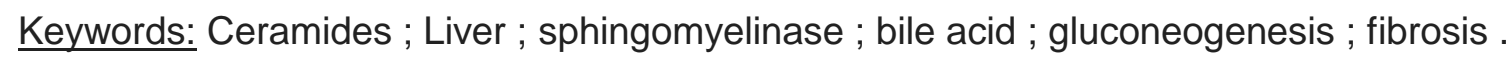




\section{Introduction}

Type 2 diabetes (T2D) impacts the lives of about 400 million people worldwide, and it is considered as a non-infectious pandemic due to the constant increase in the number of patients. The established disease is characterised by liver, fat and muscle insulin resistance, and by inadequate pancreatic beta-cells insulin secretion to counteract the insulin resistance. A specific class of lipids, namely sphingolipids, and in particular ceramides (Cer), are proposed to be important mediators of both free-fatty acid (FFA)-induced $\beta$ cell dysfunction and apoptosis, and FFA-induced insulin resistance in insulin target tissues (Poitout et Robertson 2008). Moreover, we previously showed that ceramides and dihydroceramides (dhCer) could be relevant plasma biomarkers of T2D susceptibility (Wigger et al. 2017). At the cellular level, increased concentrations of Cer promote insulin resistance by inhibiting insulin signalling pathway, and Cer can regulate autophagy, reactive oxygen species production, cell proliferation, inflammation and apoptosis (Chaurasia et Summers 2015).

In mammals, three main pathways have been described to produce sphingolipids. First, the de novo synthesis pathway starts on the cytoplasmic face of the endoplasmic reticulum (ER) with the condensation of Palmitoyl-CoA and L-Serine by serine palmitoyltransferase (SPT) to form 3-ketosphinganine. SPT is a heterodimer which consists of three subunits called SPTLC1, SPTLC2, and SPTLC3 (Hornemann, Wei, et von Eckardstein 2007). Then, ceramides are transported to the Golgi apparatus to be metabolized into more complex sphingolipids such as glucosyl-ceramides and sphingomyelin (Bellini et al. 2015). Second, the catabolic sphingomyelinase pathway leads to the degradation of sphingomyelin (SM) into ceramides by sphingomyelinases and takes place at different sub_cellular localizations (plasma membrane, mitochondria, lysosome and Golgi) ((Insausti-Urkia et al. 2020). The third pathway is called the "salvage pathway" from late endosomes/lysosomes, which generate ceramides from the breakdown of complex sphingolipids (Kitatani, Idkowiak-Baldys, et Hannun 2008). Cer are produced by ceramide synthase (CerS) through $\mathrm{N}$-acylation of a sphingoid base, both in de novo synthesis and salvage pathway. Among the different ceramide species, C18:0 and C16:0-Cer have been pointed out as apoptosis inducers which play a key role in the development of insulin resistance and steatohepatitis (Raichur et al. 2014; Pewzner-Jung et al. 2010).

The liver is a key metabolic organ which govern body energy metabolism. Thus, the liver is at the core of the T2D disease. In particular, the intrahepatic accumulation of lipids and, especially ceramides, observed in NASH syndrome (nonalcoholic steatohepatitis) strongly 
correlates with the risk of T2D (Birkenfeld et Shulman 2014). In addition, it has been demonstrated that in vivo or in vitro inhibition of SPT, using myriocin, decreases ceramides accumulation in plasma and liver and improves hepatic fibrosis, steatosis and insulin sensitivity (Kasumov et al. 2015; M. Jiang et al. 2019).

Hepatocytes, the major parenchymal cells in the liver, carry out many metabolic functions, including the production of bile acids (BA). BA are amphipathic molecules, which allow dietary lipid absorption and also act as signaling molecules through their action on nuclear receptors such as the Farnesoid $X$ receptor (FXR) or G protein-coupled bile acid receptors, such as Takeda G protein-coupled receptor 5 (TGR5). FXR is activated by chenodeoxycholic acid (CDCA) but inhibited by tauro-beta-muricholic acid (T- $\beta-M C A)$, and regulates bile acid synthesis (Goodwin et al. 2000; Sayin et al. 2013). Numerous studies have demonstrated the role of FXR signaling in glucose homeostasis and especially in hepatic glucose production (Sun, Cai, et Gonzalez 2021). Thus, beyond their detergent properties, bile acid modification could trigger metabolic disorders in an endocrine way. Interestingly, inhibition of intestinal FXR signaling prevents obesity induced by high fat diet and metabolic disease such as insulin resistance and fatty liver (C. Jiang et al. 2015). Recently, Smpd3/nSmase2 and Sptlc2 genes, involved in ceramide production, have been identified as FXR target genes (Xie et al. 2017; Wu et al. 2021). These results suggest the existence of a ceramide/FXR/BA signaling axis, which could regulate glucose and lipid metabolism.

Because of a key role of hepatic sphingolipids production in aetiology of metabolic diseases we investigated the role of de novo ceramide synthesis in the liver of mice fed with regular chow or HFD on energy homeostasis. Using the cre-lox system, we developed a mouse model to decrease ceramide de novo synthesis in liver by targeting the rate-limiting enzyme, Sptlc2, in hepatocytes. Interestingly, we found that a 50\% decrease of liver Sptlc2 expression impaired bile acid synthesis and export but also led to a defect in lipid absorption. Our results also showed that Spt/c2-deficient mice were protected against HFD-induced obesity and displayed impaired gluconeogenesis, while their liver ceramide content was increased and sphingomyelin content decreased.

Altogether, we demonstrated for the first time that Spt/c2 deficiency was associated with strong alteration in bile acid (BA) homeostasis, inhibition of FXR signalling, decreased hepatic glucose production, and progressive liver fibrosis. 


\section{Materials and methods}

\subsection{Animals}

All procedures were carried out in accordance with the ethical standards of French and European regulations (European Communities Council Directive, 86/609/EEC). Animal use and procedures were approved by the Ethics committee of the University of Paris and by the French Ministry of Research under the reference \#2016040414129137. Sptlc2fl ${ }^{f f l}$ mice (kindly given by Xian-Cheng Jiang) were crossed with $\mathrm{AlbCre}^{+/-}$mice (kindly given by Catherine Postic) to generate mice lacking Spt/c2 expression in hepatocytes Spt/c2 ${ }^{\Delta H e p}$ or littermate

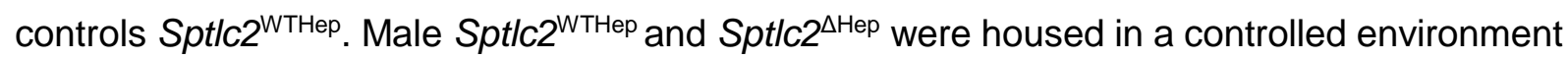
with tap water and ad libitum regular food (A04 diet, Safe) or high fat diet (HF260 diet, Safe) starting at 8 weeks of age. Mice were maintained on a 12-hour light/12-hour dark cycle and cages were enriched with tunnels. Number of mice and suffering were minimized in accordance with the 3 Rs.

\subsection{Metabolic phenotyping}

Body weight and body mass composition

Body weight was measured weekly. Body mass composition was determined by an EchoMRI900 (Echo Medical Systems).

\section{$\underline{\text { Oral glucose tolerance test }}$}

Oral glucose tolerance tests (OGTT) were performed in overnight fasting mice. A glucose solution $(2 \mathrm{~g} / \mathrm{kg}$ ) was administrated by oral gavage. Blood glucose was quantified from the tip of the tail vein with a glucose meter (Glucofix Lector, Menarini Diagnostics). Blood samples were collected from the tail vein to assay plasma insulin with a wide-range Mouse ultrasensitive Insulin ELISA Kit (catalog no. 80-INSMSU-E01, Alpco).

\section{$\underline{\text { Insulin tolerance test }}$}

To assess insulin sensitivity, insulin tolerance tests $(0.5 \mathrm{U} / \mathrm{kg}$ for HFD mice and $0,25 \mathrm{U} / \mathrm{kg}$ for $\mathrm{RC}$ mice) were performed in 5-hour-fasting mice. Mice were given an intraperitoneal injection of insulin (Novo Nordisk), and blood glucose was monitored following the same protocol as was used for OGTT.

Pyruvate and glycerol tolerance test 
To evaluate hepatic glucose production, pyruvate at $2 \mathrm{~g} / \mathrm{kg}$ (sodium pyruvate, catalog no. P5280, Sigma) or glycerol at 1 g/kg (catalog no. G5516) were injected (intraperitoneal injection) in overnight fasting mice. Blood glucose was measured every 15 or 30 minutes during 120 minutes and quantified from the tip of the tail vein with a glucose meter (Glucofix Lector, Menarini Diagnostics).

Blood ketone bodies were quantified from the tip of the tail vein with a glucose meter (Glucofix Lector, Menarini Diagnostics).

\subsection{Energy content of feces by bomb calorimeter}

Feces were collected every $24 \mathrm{~h}$ during one week, dried to constant weight $(0.001 \mathrm{~g})$ at $60^{\circ} \mathrm{C}$, and energy content of dried feces $(\mathrm{kJ} / \mathrm{g})$ was analyzed for each individual mouse using a bomb calorimeter (IKA C200, Staufen, Germany).

\subsection{Liver histology}

Liver samples were either frozen in nitrogen-cooled isopentane and stored at $-80^{\circ} \mathrm{C}$ until use or fixed in 4\% formaldehyde and embedded in paraffin. H\&E (Haemotoxylin and Eosin) and Sirius red staining were performed following standard procedures.

\section{$\underline{\text { Immunohistochemistry }}$}

Frozen Liver samples sections $10-\mu \mathrm{m}$ thick were cut with a refrigerated cryostat, collected on slides, and stored at $-80{ }^{\circ} \mathrm{C}$ until staining. Antibodies against: Ki67 (catalog no.15580, 1/500, Abcam), Gr-1 (catalog no. 550291, 1/50, BD Pharmingen) and the cholangiocyte marker cytokeratin-19 CK19 (TROMA-III, Developmental Studies Hybridoma Bank, University of lowa, $1 / 500$ ) were used. Liver sections were then incubated with secondary antibodies (Alexa fluor $1 / 500$, Molecular Probes) $30 \mathrm{~min}$ at $37^{\circ} \mathrm{C}$. Images were acquired by epifluorescence (Axioskope, Zeiss).

\subsection{Real time PCR analysis}

RNA extraction and $c D N A$ synthesis

Total RNA was isolated from the liver using RNeasy Mini kit (Qiagen). The concentration of RNA samples was ascertained by measuring optical density at $260 \mathrm{~nm}$. The integrity of RNA was confirmed by the detection of $18 \mathrm{~S}$ and $28 \mathrm{~S}$ bands after agarose-formaldehyde gel electrophoresis. The quality of RNA was verified by optical density absorption ratio OD 260nm / OD 280nm. To remove residual DNA contamination, the RNA samples were treated with DNAse RNAse-free (Qiagen) and purified with Rneasy mini column (Qiagen). $1 \mu \mathrm{g}$ of total RNA 
from each sample was reverse transcribed with $40 \mathrm{U}$ of M-MLV Reverse Transcriptase (Invitrogen, life technologies) using random hexamer primers.

\section{Real-time PCR using SYBR-Green chemistry}

Real time quantitative PCR amplification reaction were carried out in a LightCycler 480 detection system (Roche) using the LightCycler FastStart DNA Master plus SYBR Green I kit (Catalog $n^{\circ} 03515869001$, Roche). 40ng of reverse transcribed RNA was used as template for each reaction. All reactions were carried out in duplicate with no template control. The PCR conditions were: $95^{\circ} \mathrm{C}$ for $10 \mathrm{~min}$, followed by 40 cycles of $95^{\circ} \mathrm{C}$ for $10 \mathrm{sec}, 60^{\circ} \mathrm{C}$ for $10 \mathrm{sec}$ and $72^{\circ} \mathrm{C}$ for $10 \mathrm{sec}$. To compare target level, relative quantification was performed using the $2^{-\Delta \Delta \mathrm{Ct}}$ methods. The relative abundances of the involved genes were calculated by normalizing their level to those of Cyclophilin A (CycloA), 18S ribosomal RNA (18S) and TATA-binding protein $(T b p)$. The primers were derived from mouse sequences and are listed in supplemental methods.

\subsection{Western blot analysis}

Samples of frozen mouse liver were homogenized in lysis and extraction buffer (RIPA lysis and extraction buffer, Thermofisher Scientific, catalog $\left.n^{\circ} 89900\right)$ containing protease inhibitors (protease inhibitor cocktails tablets, Roche, catalog $n^{\circ}$ 48047900). After high-speed shaking in TissueLyserll (Quiagen) with stainless steel beads, the homogenate was centrifuged at $15000 \mathrm{rpm}$ for $15 \mathrm{~min}$ at $4{ }^{\circ} \mathrm{C}$, and the supernatant was collected in a new tube. After appropriate quantitative analysis (protein quantitation kit, Interchim), equal amounts of the protein samples $(20 \mu \mathrm{g}$ of liver extracts) were resuspended in Laemmli sample buffer (Thermofisher Scientific, catalog $n^{\circ} 39000$ ) and separated in an 4\%-20\% sodium dodecylsulfate (SDS) polyacrylamide gel system (Biorad). After transfer, the nitrocellulose membranes were incubated with specific antibodies overnight at $4{ }^{\circ} \mathrm{C}$ and then with the secondary antibody conjugated with peroxidase for $1 \mathrm{~h}$ at RT. The primary antibodies used were the following: anti-cleaved Casp3 (Cell Signalling, catalog $n^{\circ} 9661,1 / 500$ ), anti-Smpd3 (Merck clone 14G5.1, 1/2000), and anti-Cyclophilin A (Cell Signalling, catalog $n^{\circ} 51418$, 1/2000). Immunoreactivity was detected with an ECL Western Blotting Analysis System (Amersham) and acquired and analyzed using Amersham ${ }^{\mathrm{TM}}$ Image 6000 and the Image $\mathrm{J}$ software (LiCor Biosciences).

\subsection{Quantification of phosphorylated (Ser473) Akt and total Akt}

Protein extraction was carried out from liver, adipose tissue and muscle was carried out following the same protocol as was used for western blot analysis. Quantitative determination 
of phospho-Akt (Ser473) and total Akt was assessed by a Meso Scale Discovery (MSD) multispot electrochemiluminescence immunoassay system (Phospho(Ser473)/Total Akt Whole Cell Lysate Kit, catalog ${ }^{\circ}$ K15100D) according to manufacturer's instructions.

\subsection{Biochemical analyses}

Liver triglycerides were measured using triglyceride determination kit from Sigma (catalog $\mathrm{n}^{\circ} \mathrm{TR} 0100$ ) according to the manufacturer's instructions. Liver glycogen content was measured using amyloglucosidase enzyme (Sigma-Aldrich, catalog $\left.n^{\circ} \mathrm{A} 7095\right)$ assay according to Roehring and Allred method (Roehrig et Allred 1974). Glucose was then determined using Glucose GOD-PAP kit (Biolabo, catalog $n^{\circ} 87109$ ). Plasma concentrations of non-esterified fatty acid (NEFA), cholesterol, HDL cholesterol, alanine amino-transferase (ALAT), aspartate amino-transferase (ASAT), direct bilirubin and alkaline phosphatase were determined using an automated Monarch device (CEFI, IFR02, Paris, France) as described previously (Viollet et al. 2003).

\subsection{Isolation of primary mouse hepatocytes}

Hepatocytes from 45 day-old Spt/c2 $2^{\mathrm{WTHep}}$ and $S p t / c 2^{\Delta \mathrm{Hep}}$ mice were isolated as previously described (Besnard et al. 2016).

\subsection{Measurement of glucose production from isolated hepatocytes}

Primary mouse hepatocytes were cultured in BD BioCoat ${ }^{\mathrm{TM}}$ Collagen I 6-well plates (1-1.5 $\mathrm{x}$ $10^{6}$ cells per well) in William's medium (Gibco catalog $n^{\circ} 12551032$ ) supplemented with $5 \%$ FBS, penicillin (100 units $/ \mathrm{ml})$ and streptomycin $(100 \mu \mathrm{g} / \mathrm{ml})$. The day after primary hepatocytes isolation, cells were washed two times with $\mathrm{PBS}+/+$ and medium was replaced with glucosefree DMEM (Sigma-Aldrich, catalog ${ }^{\circ}$ D5030) without phenol red supplemented with $2 \mathrm{mM} \mathrm{L-}$ glutamine, $15 \mathrm{mM}$ HEPES (Gibco, catalog $\left.\mathrm{n}^{\circ} 15630-056\right)$, 5\% penicillin (100 units $/ \mathrm{ml}$ ) and streptomycin $(100 \mu \mathrm{g} / \mathrm{ml})$ and in presence or absence (negative control) of $20 \mathrm{mM}$ sodium lactate (Sigma-Aldrich catalog $n^{\circ}$ L7022), 1,5 mM sodium pyruvate (Gibco catalog $n^{\circ} 11360$ 070) and pCPT-cAMPC (Sigma-Aldrich, catalog $n^{\circ} \mathrm{C} 3912$ ) at $100 \mu \mathrm{M}$ as positive control. Hepatocytes were then incubated for $2 \mathrm{hr}$ at $37^{\circ} \mathrm{C}$ and $150 \mathrm{ul}$ of cell medium was collected $2 \mathrm{~h}$ and $3 \mathrm{~h}$ after stimulation for glucose measurement. The glucose content of the supernatant was measured using hexokinase (Sigma-Aldrich, catalog $n^{\circ}$ H4502) and glucose-6-phosphatase enzyme (Sigma-Aldrich, catalog $\left.n^{\circ} \mathrm{G} 8404\right)$.

\subsection{G6pase activity assay and liver G6P content measurement}

G6Pase activity was assayed in liver homogenates for $10 \mathrm{mn}$ at $30^{\circ} \mathrm{C}$ at $\mathrm{pH} 7.3$ in the presence of a saturating glucose-6-phosphate concentration of $20 \mathrm{mM}$ as described in (Mithieux et al. 
2004; Rajas et al. 1999). Hepatic glucose-6-phosphatase (G6P) determinations were carried out as previously described (Penhoat et al. 2014).

\subsection{Quantification of sphingolipids}

All solvents used were LC-MS grade and purchased from Biosolve (Valkenswaard, Netherlands). Standard compounds were obtained from Sigma Aldrich (Saint-Quentin Fallavier, France). Individual stock solutions $(2.5 \mathrm{mmol} / \mathrm{L})$ of sphingolipids (sphingosine-1phosphate [S1P], ceramides [Cer], hexosyl ceramides [HexoCer], lactosyl ceramides [LactoCer] and sphingomyelins [SM]), and exogenous S1P (d17:1), Cer (18:1/17:0) and SM (18:1/17:0) were prepared in isopropanol. A pool of reference standard solutions including S1P, 6 Cer species, 3 HexoCer species, 3 LactoCer species and 6 SM species (Supplemental Table S1) was prepared in isopropanol and then serially diluted to obtain seven standard solutions ranging from $1-1000 \mathrm{nmol} / \mathrm{L}$ for S1P, 5-5000 nmol/L for Cer, HexoCer and LactoCer, and 0.1-100 $\mu \mathrm{mol} / \mathrm{L}$ for $S M$. Liver tissues samples were weighted and diluted $(0.1 \mathrm{~g} / \mathrm{mL})$ in PBS before homogenization. Standard solutions, liver homogenates and plasma samples (10 $\mu \mathrm{L})$ were then extracted with $500 \mu \mathrm{L}$ of methanol/chloroform mixture $(2: 1 ; \mathrm{v}: \mathrm{v})$ containing exogenous internal standards (IS) at $0.5 \mu \mathrm{mol} / \mathrm{L}, 1 \mu \mathrm{mol} / \mathrm{L}$ and $5 \mu \mathrm{mol} / \mathrm{L}$ for S1P (17:1), Cer (18:1/17:0) and SM (18:1/17:0), respectively. Samples were mixed and centrifuged for $10 \mathrm{~min}$ at $20,000 \times \mathrm{g}\left(4^{\circ} \mathrm{C}\right)$. The supernatants were dried under a gentle stream of nitrogen. Dried samples were finally solubilized in $100 \mu \mathrm{L}$ of methanol prior liquid chromatography-tandem mass spectrometry (LC-MS/MS) injection.

Sphingolipid concentrations were determined in plasma and liver tissue by LC-MS/MS on a Xevo ${ }^{\circledR}$ Triple-Quadrupole mass spectrometer with an electrospray ionization interface equipped with an Acquity H-Class ${ }^{\circledR}$ UPLC $^{T M}$ device (Waters Corporation, Milford, MA, USA). Samples $(10 \mu \mathrm{L})$ were injected onto an Acquity BEH-C ${ }_{18}$ column $(1.7 \mu \mathrm{m} ; 2.1 \times 50 \mathrm{~mm}$, Waters Corporation) held at $60^{\circ} \mathrm{C}$, and compounds were separated with a linear gradient of mobile phase B (50\% acetonitrile, $50 \%$ isopropanol containing $0.1 \%$ formic acid and $10 \mathrm{mmol} / \mathrm{L}$ ammonium formate) in mobile phase A (5\% acetonitrile, $95 \%$ water containing $0.1 \%$ formic acid and $10 \mathrm{mmol} / \mathrm{L}$ ammonium formate) at a flow rate of $400 \mu \mathrm{L} / \mathrm{min}$. Mobile phase $B$ was linearly increased from $40 \%$ to $99 \%$ for $4 \mathrm{~min}$, kept constant for $1.5 \mathrm{~min}$, returned to the initial condition over $0.5 \mathrm{~min}$, and kept constant for $2 \mathrm{~min}$ before the next injection. Target compounds were then detected by the mass spectrometer with the electrospray interface operating in the positive ion mode (capillary voltage, $3 \mathrm{kV}$; desolvatation gas $\left(\mathrm{N}_{2}\right)$ flow, $650 \mathrm{~L} / \mathrm{h}$; desolvatation gas temperature, $350^{\circ} \mathrm{C}$; source temperature, $120^{\circ} \mathrm{C}$ ). The multiple reaction monitoring mode was applied for MS/MS detection as detailed in Supplemental Table 1. Chromatographic peak area ratios between sphingolipids and IS constituted the detector responses. Standard solutions were used to plot the calibration curves for quantification. The assay linearity was 
expressed by the mean $R^{2}$, which was greater than 0.996 for all compounds (linear regression, $1 / x$ weighting, origin excluded). Data acquisition and analyses were performed with MassLynx and TargetLynx version 4.1 softwares, respectively (Waters Corporation).

\subsection{Bile acids measurements}

Bile acids measurements were performed on mouse bile, plasma, liver and feces by highperformance liquid chromatography-tandem mass spectrometry as described in (Péan et al. 2013; Bidault-Jourdainne, Merlen et al, 2020). The hydrophobicity index reflects bile acids hydrophobicity, taking into account the retention time (RT) of different bile acids on a C18 column with a gradient of methanol; the LCA has the highest retention time, the TUDCA-3S has the lowest.

\subsection{Statistical Analysis}

Data are expressed as means \pm SEM. Statistical analysis was performed using Student's $t$ test, Mann-Whitney U test or two-way ANOVA followed by two-by-two comparisons using Bonferroni's post hoc test (GraphPad Prism 6 Software, La Jolla CA, USA). Differences were considered significant at $p<0.05$. 


\section{Results}

\subsection{Genetic deletion of Sptlc2 in hepatocytes increases a selective ceramide content in the liver but decreases sphingomyelin content associated with an upregulation of}

\section{nSmase2}

Sptlc2lox/lox mice were crossed with heterozygous mice which expressed the protein CRE driven by the albumin promoter (Figure S1.A). As previously shown (Li et al. 2016), Sptlc2 hepatic mRNA level was reduced to $60 \%$ of WT level (Figure S1.B), consistent with the fact that hepatocytes represent about 70\% of all liver cells (Si-Tayeb, Lemaigre, et Duncan 2010). As measured by quantitative-PCR, Spt/c2 deficiency induces overexpression of Desg1, CerS5 and CerS6 genes but had no effect on the transcription of other enzymes involved in ceramide de novo synthesis pathway (Figure S1.C). It has been demonstrated that in vivo or in vitro pharmacological inhibition of SPT, using myriocin, decreases ceramides accumulation in plasma and liver and improves hepatic fibrosis, steatosis and insulin sensitivity (Kasumov et al. 2015; M. Jiang et al. 2019; Aji et al. 2020). However, in our genetic model, Spt/c2 deficiency in hepatocytes leads to $\mathrm{C} 16: 0, \mathrm{C} 18: 0$ and C24:1-ceramide accumulation in the liver and plasma in mice fed with regular chow (RC) or high fat diet (HFD) (Figure 1.A-D, Figure S1.DE and S2.). Interestingly, C16:0 and C18:0 -ceramides, are known as apoptosis inducers and insulin resistance mediators (Hartmann et al. 2012; Raichur et al. 2019). Quantification of ceramides in isolated hepatocytes from Spt/c2 $2^{\Delta \mathrm{Hep}}$ mice and their littermate controls confirmed C16:0-ceramide upregulation (Figure S1.F, S1.G and S1.H). Interestingly, CerS5 and CerS6 which catalyze $\mathrm{C} 16: 0$ and $\mathrm{C} 18: 0$ ceramides synthesis are up regulated in liver of Knock Down (KD) mice upon HFD (Figure S1.C).

On the contrary, sphingomyelin content was reduced in the liver of Spt/c2 Knock-Down (KD) mice, at the expense of ceramide (Figure 1.A and 1.B). Indeed, the ratio ceramide/sphingomyelin is up to 3 times more elevated for each species in the liver of Sptlc $2^{\Delta \mathrm{Hep}}$ mice (Figure 1.E and 1.F). These data suggest that the sphingomyelinase (SMase) pathway from hydrolysis of sphingomyelin (SM) is over-activated to produce ceramides. SMase pathway takes place at different sub-cellular localizations and involves different SMases depending on the $\mathrm{pH}$ of the organelles. Then, neutral SMase occurs at the plasma membrane while acid SMase occurs into the lysosome. We measured the expression level of the Smases and we found that mRNA level of nSMase 2 is dramatically elevated in the liver of KD mice, independently of the diet, compared to the littermate controls (Figure 1.G and 1.H). Moreover, nSmase2 protein detection by western blotting confirms this result (Figure 1.I). 
Overall, these data suggest a compensatory mechanism through the SMase pathway to maintain an elevated ceramide content in the liver despite the inhibition of de novo synthesis pathway.

\subsection{Spt/c2 deficiency impairs bile acid synthesis and transport and leads to a defect in lipids absorption.}

We placed Spt/c2 ${ }^{\Delta \mathrm{Hep}}$ mice and their littermate controls on HFD for 8 weeks in an attempt to induce obesity and insulin resistance. As shown in Figure 2.A, starting from week 4, mutant mice have significantly gained less body weight than control animals, despite similar food intake (Figure 2.C). Mass composition analysis revealed a decreased fat mass in KD mice compared to WT mice upon RC and HFD (Figure 2.D). The analysis of energy expenditure and thermogenesis failed to explain the body weight difference (data not shown). However, energetic density of faeces collected every $24 \mathrm{~h}$ during 15 days is increased in KD mice (Figure 2.E), and we show that Spt/c2 deficiency in hepatocytes impaired triglycerides absorption. Indeed, triglycerides amount is reduced in the plasma of Spt/c2 ${ }^{\Delta \mathrm{Hep}}$ mice up to 6 hours after an oral olive oil charge (Figure 2.F). These results suggest that Sptlc2 ${ }^{\Delta \mathrm{Hep}}$ mice exhibit a defect of lipids absorption leading to resistance to body weight gain normally induced by HFD.

As modifications of BA composition, synthesis and transport could impair lipid absorption (Holt 1972), we measured BA composition and concentration in the gallbladder, liver, ileum and plasma and showed that KD mice compared to WT mice exhibited substantially elevated BA concentrations in liver, gallbladder and plasma (Figure 2.G, Figure S3.A). Moreover, BA composition was strongly modified between the two groups of mice, independently of the diet (Figure 2.H, Figure S3.B). A dramatic increase in tauro muricholic acid (T-MCA) was observed in mutant mice (Figure 1.J, Figure S3.C) while secondary BA were almost absent (Figure 2.H, Figure S3.B). In line with these data, the hydrophobicity index of BA, was decreased in liver and gallbladder from Spt/c2 ${ }^{\Delta \mathrm{Hep}}$ as compared with WT mice (Figure 2.I, Figure S3.D). Elevated plasma bile acids were associated with severe jaundice in mutant mice, along with moderate cytolysis and strong elevation of ALP and Bilirubin (Figure 2.K and Table 1.), consistent with cholestasis, as reported (Li et al. 2016).

Interestingly in this cholestatic context, mutant mice exhibited disrupted localization of CK19 immunostaining (a marker of cholangiocytes) through hepatic lobules (Figure 2.L), suggesting that cholangiocyte injury occurred in these mice and may contribute to bile flow impairment, i.e. to cholestasis. Furthermore, we measured mRNA levels of bile acid transporters and the main enzymes involved in bile acids synthesis. Expression level of Cyp8ab1, which allows CA synthesis, and Cyp27a1, involved in the alternative pathway were both reduced in Spt/c2 ${ }^{\Delta \mathrm{Hep}}$ mice (Figure 2.M). We also found that mRNA level of the BA transporter Bile salt export pump 
(Bsep), which allows BA excretion from hepatocytes to bile canaliculus and the transporter Sodium taurocholate cotransporting polypeptide (Ntcp), a sodium-dependent transporter expressed on the basolateral membrane of hepatocytes, responsible for BA uptake from sinusoids, are both decreased in the liver of KD mice (Figure 2.N). The significant drop of Ntcp expression could be part of an adaptive response to BA accumulation in hepatocytes as already well described (Zollner et al. 2001). Consistent with the dramatic increase in T-MCA, a BA reported as an FXR antagonist (Sayin et al. 2013), a significantly decreased expression in the FXR target genes small heterodimer partner (Shp) and fibroblast growth factor 15 (Fgf15) was observed in both ileum and liver from Sptlc2 ${ }^{\Delta \mathrm{Hep}}$ as compared with WT mice (Figure 2.0 and 2.P).

\subsection{Genetic deletion of Spt/c2 impairs hepatic glucose production and storage in an insulin-independent manner.}

The liver is at the core of glucose metabolism: it produces glucose from the breakdown of glycogen, or through gluconeogenesis from lactate, pyruvate, glycerol and amino acids. These pathways are essential for normoglycemia maintenance. Interestingly, decreased level of hepatic Spt/c2 prevents glucose intolerance in mice challenged with HFD (Figure 3.A, 3.B and 3.C). De novo glucose production from pyruvate (Figure 3.D, 3.E and 3.F) and glycerol (Figure 3.G, 3.H and 3.I) are disrupted both in KD mice fed with HFD or regular chow. However, glucose production from glutamine, the preferential substrate for intestinal gluconeogenesis, is not affected in KD mice (Figure S4.C). These results are supported by glucose production measurements on isolated hepatocytes (Figure 3.J). Two and three hours after hepatocyte stimulation induced by medium without glucose containing pyruvate and glycerol, we detected a tendency of a reduced glucose production in isolated Spt/c2 $2^{\Delta \mathrm{Hep}}$ hepatocytes. Indeed, hepatic Sptlc2 deficiency decreases glucose-6-phosphatase (G6pase) and pyruvate carboxylase $(P C)$ expression levels, two enzymes involved in glycogenesis and/or gluconeogenesis (Figure 3.K). Furthermore, G6pase activity and glucose-6-phosphate content were decreased in mutant mice liver (Figure 3.L). Reduced liver glycogen content was also observed in Sptlc2 ${ }^{\Delta \mathrm{Hep}}$ mice at fed state or at $5 \mathrm{~h}$ fasted state, as compared with WT mice (Figure 3.M).

In order to evaluate the role of insulin on hepatic glucose production in our model lacking Sptlc2 expression in hepatocytes, we measured insulin secretion, during the course of a glucose tolerance test, and insulin sensitivity. Hepatic deletion of Spt/c2 did not impair insulin secretion or systemic insulin sensitivity (Figure 3.N, 3.0 and 3.P) but, consistent with the previous results, $5 \mathrm{~h}$ fasting glycemia is reduced in KD mice (Figure S4.A and S4.B). Moreover, as expected based on $\mathrm{C} 16: 0$ and $\mathrm{C} 18: 0$-cer accumulation in the liver, activation by 
phosphorylation of Akt/PKB (Protein Kinase B), a central mediator of insulin's anabolic effects is reduced in the liver of mutant mice (Figure 3.Q). Systemic insulin sensitivity seems to be compensated by an enhanced Akt/PKb phosphorylation in the muscle (Extensor Digitorum Longus, EDL). Altogether, these results demonstrated that hepatic Spt/c2 deficiency impairs hepatic glucose production in an insulin-independent manner.

Furthermore, fatty acid synthase (Fas) mRNA level and ketone bodies content in liver at $24 \mathrm{~h}$ fasted are decreased in Spt/c2 ${ }^{\Delta \mathrm{Hep}}$ mice (Figure S4), indicating both disruption in fatty acid synthesis and degradation. Together with our previous observation (Figure 2. and supplemental table 2), these data suggest that hepatic Spt/c2 impairs lipid uptake and metabolism in the liver.

\subsection{Hepatic Spt/c2 deficiency leads to liver fibrosis, inflammation and apoptosis}

Based on our data, we next asked whether modulating ceramide levels could lead to liver damage. Sirius red and H\&E liver section staining revealed that Sptlc2 $\triangle$ Hep mice exhibited necrosis, inflammatory infiltration and fibrosis, increasing progressively with age (Figure 4.A). At 20 days, bile infarcts (i.e. necrotic cell clusters characteristic of bile acid overload), were observed in Sptlc2 ${ }^{\Delta \mathrm{Hep}}$ but not WT livers, without any significant fibrosis. These results were associated with an increased liver to body weight ratio, without triglyceride accumulation, as compared to the littermate controls (Figure 4.B and 4.C).

To further investigate cellular mechanisms that trigger liver disorders, we assessed inflammatory response activation through the presence of Granulocyte receptor-1 (Gr-1) positive cells in frozen liver tissues section. Gr-1 is expressed in monocytes, neutrophils, macrophages, plasmacytoid dendritic cells, and T cells. In line with H\&E staining, as shown in Figure 4.D, inflammatory Gr1 positive cells were strongly increased in KD mice liver sections as compared with WT mice. Also, hepatic mRNAs encoded by tumor necrosis alpha ( TNFa)a proinflammatory cytokine also involved in apoptosis- and its TNF receptor 1 (TNFR-1), were significantly elevated in mice lacking expression of Sptlc2 in the liver (Figure 4.E).

Western blot analysis revealed increased cleaved Casp3 content in the liver from HFD-fed Sptlc2 $2^{\Delta \mathrm{Hep}}$ as compared with WT mice (Figure 4.F). Moreover, in Sptlc2 ${ }^{\Delta \mathrm{Hep}}$ mice, genes belonging to B-cell lymphoma 2 (Bc/2) family (apoptosis regulators), were significantly modulated. BCl-2 and Bcl-2-associated X protein (Bax), respectively anti-apoptotic and proapoptotic molecules, were up-regulated, indicating apoptosis modulation in KD mice upon HFD (Figure 4.H). In agreement with these results, Ki67 immunostaining (a proliferation marker), revealed a dramatic increase of cell proliferation in mutant as compared with WT mice, demonstrating a high apoptosis/proliferation rate in the liver of mice lacking SPTLC2 (Figure 4.F). Interestingly, this Spt/c2 ${ }^{\Delta \mathrm{Hep}}$ phenotype was not associated with any ER stress, since 
Gpr78 and Chop expression in liver were not affected by Sptlc2 deficiency (Figure S5.A and $\underline{\text { S5.B. }}$.

Altogether, these results showed that hepatic Sptlc2 disruption led to early inflammatory liver injury, with progressive development of liver fibrosis with age, in association with abnormally high rates of apoptosis and proliferation. 


\section{Discussion}

We demonstrate for the first time a potential compensatory mechanism to regulate hepatic ceramides content from sphingomyelin hydrolysis, and highlight the role of hepatic sphingolipid modulation on hepatic glucose production through bile acid composition changes.

We investigated the role of ceramide de novo synthesis in liver on energy homeostasis in mice upon regular chow (RC) or high fat diet (HFD). Interestingly, genetic deletion in hepatocytes of the rate-limiting enzyme of ceramide de novo synthesis, Sptlc2, does not reduce total intrahepatic and plasmatic ceramides level, and even increases liver ceramide content in HFD fed mice. However, we show a significant decrease of the C22:0-cer specie in liver, and both C22:0-cer and C24:0-cer diminution is evidenced in primary hepatocytes. These ceramide species are the most abundant in liver, partially because of the high expression level of CerS2 involved in C22:0 and C24:0 formation from dihydrosphingosine ( $§$ Laviad et al., 2008). Thus, in our model, Spt/c2 deficiency reduces ceramide production from de novo synthesis for the benefit of another production pathway. Indeed, neutral sphingomyelinase 2 (nSMase2) mRNA and protein level are up-regulated in $S p t / c 2^{\Delta H e p}$ mice, suggesting over-activation of the sphingomyelinase pathway at plasma membrane. Our data suggest for the first time to our knowledge a compensatory mechanism to maintain (and even to increase) ceramide production though the dramatic upregulation of $n S m a s e 2$. As a direct regulatory interaction between SPTLC2 and nSmase2 is unlikely due to different intracellular localization, one could speculate that the decreased utilization of serine and palmitoyl-CoA (SPTLC2 substrates) led to a modulation of $n S m a s e 2$ transcription and synthesis. Indeed, it has been shown that decreased ceramides de novo synthesis leads to a diminution in membrane sphingomyelins (SM), a situation which compromises membrane integrity and lipid rafts distribution (Li et al. 2016). Although the fine mechanisms leading to an overproduction of ceramides remain unclear, these data suggest that ceramides are essential components for the cell and that an attempt to alter their de novo synthesis would trigger a cellular response to avoid a drop in ceramide content, at the expense of SM concentration, a situation occurring in liver that also leads to important changes in plasma lipids profile.

Interestingly, acute reduction of Spt/c2 expression or activity using adenovirus targeting Sptlc2 in liver, or pharmacological inhibitors such as myriocin, doesn't induce ceramide accumulation (§ Zabielski et al., 2019 ; Li et al., 2009) in contrast to chronic Sptlc2 deletion using genetic approach which led to ceramide and especially $\mathrm{C} 16: 0$ increase in liver and adipose tissue (§ Li et al., 2016 ; Lee et al., 2017). In the liver, authors have shown that specific deletion of Spt/c2 induces sphingomyelin reduction and ceramide accumulation in plasma membrane of hepatocytes, supporting the hypothesis of a compensatory mechanism through over-activation 
of SM hydrolysis at plasma membrane (§ Li et al., 2016). In our hands, hepatic C16:0ceramides are dramatically increased in mutant mice and these ceramides species are well described as apoptotic inducers and inflammation triggers (§ Grösch et al., 2012). Numerous studies have shown that induction of $n S m a s e 2$ expression caused $\mathrm{C} 16: 0$-ceramides accumulation, in the liver or in ileum (§ Matsubara et al., 2011 ; Wu et al., 2021). In our model, C16:0-ceramide accumulation could not be directly linked to the result of SM hydrolysis because an increased concentration of hepatic C16:0 SM is observed in Sptlc2 deficient mice fed HFD, along with the up-regulation of CerS5 and CerS6, which allow ceramide C16:0 and C18:0 formation from dihydrosphingosine. However, CerS5 and CerS6 substrates are likely provided by SM hydrolysis and ceramidase action (§ He et al., 2003). In addition, numerous studies show that $n$ Smase 2 activation induces apoptosis through different signalling pathways leading to caspase 3 (Casp3) cleavage (§ Levy et al., 2006 ; Abadie et al., 2002 ; Shamsedinne et al., 2014 ), consistently with our findings in Sptlc2 ${ }^{\Delta \mathrm{Hep}}$ mice under HFD.

We also showed that the modulation of sphingolipid metabolism in liver leads to a strong alteration of liver homeostasis, in particular we evidenced an impairment of bile acid (BA) pool composition associated with a defect in intestinal lipid absorption.

BA facilitate dietary lipids uptake in the intestine and also act as signalling molecules through their action on membrane or nuclear receptors such as FXR or TGR5. Our findings show that primary BA, especially tauro-beta-muricholic acid (T-BMCA), are increased in Sptlc2 ${ }^{\triangle \mathrm{Hep}}$ mice and that secondary BA are almost absent. After excretion in duodenum, primary BA are converted into secondary BA by the gut microbiota. Secondary BA, which are more hydrophobic than primary BA, are essential for intestinal lipid absorption (§ Woollett et al., 2004). Accordingly, Sptlc2 $\triangle$ Hep mice exhibit lower triglycerides absorption and increased energy density in feces. Taking together, these data are consistent with a full resistance to high-fat induced obesity in Spt/c2 ${ }^{\Delta \mathrm{Hep}}$ mice due to altered BA-dependent lipid absorption in the intestine. Of interest, food intake is similar in Spt/c2 ${ }^{\Delta \mathrm{Hep}}$ compared to the controls, supporting post-ingestive events rather than nervous perturbation of food behavior.

Sptlc2 ${ }^{\Delta \mathrm{Hep}}$ mice also displayed jaundice as reported ( $\S \mathrm{Li}$ et al., 2016b). Although precise mechanisms of cholestasis development in this model remain to be fully deciphered, our data suggest that in the lack of Sptlc2 in hepatocytes, intrahepatic bile ducts were disorganized, a feature that likely contributed to bile flow impairment. How cholangiocytes would be injured in these mice remains to be elucidated, but we speculate that the accumulation of proinflammatory ceramides observed in Sptlc2 ${ }^{\Delta \mathrm{Hep}}$ mice might contribute to biliary epithelial damage. 
Numerous actors allow the regulation of bile acid metabolism, among them FXR, a nuclear transcription factor also involved in glucose homeostasis. Indeed, in vitro or in vivo modulation of FXR pathway regulates gluconeogenesis in rodent. Thus, in vitro, FXR activation induces Pepck expression and FOXO1 activation (Stayrook et al. 2005) ; in vivo, FXR KO mice display lower Pepck expression and a decreased glucose production (§ Duran-Sandoval et al., 2005).

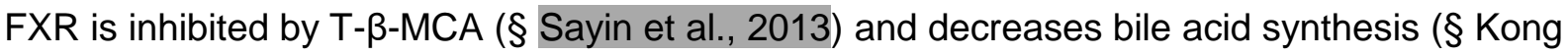
et al., 2012). Consistent with the dramatic increase of T- $\beta-M C A$, we show that genetic deletion of hepatic Sptlc2 inhibits expression of FXR signaling pathway. In addition, Spt/c2 ${ }^{\Delta \text { Hep }}$ mice have a defect in hepatic production of glucose from pyruvate and glycerol, which probably explains, at least in part, the better glucose tolerance under HF, while insulin secretion and sensitivity are similar to their respective controls. Of note, $5 \mathrm{~h}$ fasting glycemia is reduced in Spt/c2 ${ }^{\Delta \mathrm{Hep}}$ mice. These traits are unlikely linked to a liver-specific increase in insulin sensitivity, while ceramides, well-known inducers of insulin resistance, accumulates in liver. Consistently, glycogen content is reduced, and phosphorylation of Akt/ protein kinase B (PKB), a main mediator of insulin anabolic effects is reduced in the liver of mutant mice, while systemic insulin sensitivity seems to be compensated by an enhanced Akt/Pkb phosphorylation in the muscle (extenseur digitorium longus muscle). Altogether, these results demonstrated that hepatic Sptlc2 deficiency impaired hepatic glucose production in an insulin independent way.

Conversely, Spt/c2 $2^{\Delta \mathrm{Hep}}$ mice have lower hepatic glucose-6-phosphate content (consistent with their glucose production defect) which could play a causal role in the modulation of the $A B$ pool. Indeed, Hoogerland et al. put in evidence that G6P stimulates Cyp8b1 expression through activation of the transcription factor ChREBP (§ Hoogerland et al., 2019). The drop of G6P could thus explain the diminished CA production and Cyp8b1 down-expression in Spt/c2 ${ }^{\Delta \mathrm{Hep}}$ mice, as an indirect mediator of sphingolipids metabolism modulation.

Thus, we put in evidence a cascade of events, from SPTLC2 deficiency to the over expression of $\mathrm{nSmase} 2$ and the changes in BA profile which led to liver damage: liver cells organisation is altered, we evidenced a cholestasis, a fibrosis, a defect in glucose production (independent from insulin) and an increased apoptosis/proliferation rate. However, despite the jaundice, mice health condition is correct under RC and, interestingly, they are protected against highfat diet induced obesity and glucose intolerance. These data suggest a new role of nSMase2, which could be a target for decreasing hepatic glucose production, when activated. Recently, it has been shown that $n S m a s e 2$ is a target gene of FXR ( $\$$ Wu et al., 2021 ; Xie et al., 2017). Although Fxr and nSmase2 vary in opposite ways in our model, our findings suggest that nSMase2 may exert a negative feedback on FXR expression, probably through its products, ceramides. 
We also evidenced a previously undescribed age-related effect of SPTLC2 deficiency that favors hepatic fibrogenesis. Although precise mechanisms remain to be investigated, the decreased amount of membrane SMs in the lack of hepatic SPTLC2 (§ Li et al., 2016b), associated with a reduction of membrane lipids rafts, may favour beta catenin translocation to the nucleus and contribute to the onset of fibrogenesis (Ge et al. 2014) (§ Li et al., 2016b). Moreover, several studies showed that ceramides themselves are involved in hepatic fibrogenesis, and in the setting of NASH disease (revues: Ishay et al 2020; Simon et al, 2019). In particular, it has been shown in a mouse NASH model that a decrease in hepatic ceramide content (through Fgf19 treatment that modulates BA metabolism) rescued from hepatic fibrosis, TG accumulation and liver injury ( § Zhou et al., 2017). Interestingly, in our model we demonstrated that the hepatic glucose production defect was already present in Sptlc2 $\Delta \mathrm{Hep}$ mice aged $45 \mathrm{~d}$ before the neat onset of fibrosis and altogether our results suggest that hepatic defect of glucose production is independent of fibrosis development over time.

In conclusion, our findings suggest a compensatory mechanism for ceramides production in liver, and highlight a link between sphingolipid production, BA metabolism, and glucose production in an insulin-independent way. The nuclear receptor FXR, whose activation is controlled by BAs, and which is implicated in both BA homeostasis and energy metabolism is likely to be at the core of a Sphingolipids/BAs/Neoglucogenesis axis. 


\section{References}

Aji, Gulibositan, Yu Huang, Mei Li Ng, Wei Wang, Tian Lan, Min Li, Yufei Li, et al. 2020. « Regulation of Hepatic Insulin Signaling and Glucose Homeostasis by Sphingosine Kinase 2 ». Proceedings of the National Academy of Sciences 117 (39): 24434-42. https://doi.org/10.1073/pnas.2007856117.

Bellini, Lara, Mélanie Campana, Rana Mahfouz, Aurélie Carlier, Julien Véret, Christophe Magnan, Eric Hajduch, et Hervé Le Stunff. 2015. "Targeting Sphingolipid Metabolism in the Treatment of Obesity/Type 2 Diabetes ». Expert Opinion on Therapeutic Targets 19 (8): 1037-50. https://doi.org/10.1517/14728222.2015.1028359.

Besnard, Aurore, Julien Gautherot, Boris Julien, Ali Tebbi, Isabelle Garcin, Isabelle Doignon, Noémie Péan, et al. 2016. « The P2X4 Purinergic Receptor Impacts Liver Regeneration after Partial Hepatectomy in Mice through the Regulation of Biliary Homeostasis ». Hepatology (Baltimore, Md.) 64 (3): 941-53. https://doi.org/10.1002/hep.28675.

Birkenfeld, Andreas L., et Gerald I. Shulman. 2014. « Nonalcoholic Fatty Liver Disease, Hepatic Insulin Resistance, and Type 2 Diabetes ». Hepatology (Baltimore, Md.) 59 (2): 713-23. https://doi.org/10.1002/hep.26672.

Chaurasia, Bhagirath, et Scott A. Summers. 2015. «Ceramides - Lipotoxic Inducers of Metabolic Disorders ». Trends in Endocrinology \& Metabolism 26 (10): 538-50. https://doi.org/10.1016/j.tem.2015.07.006.

Duran-Sandoval, Daniel, Bertrand Cariou, Fredéric Percevault, Nathalie Hennuyer, Aldo Grefhorst, Theo H. van Dijk, Frank J. Gonzalez, Jean-Charles Fruchart, Folkert Kuipers, et Bart Staels. 2005. « The Farnesoid X Receptor Modulates Hepatic Carbohydrate Metabolism during the Fasting-Refeeding Transition ». The Journal of Biological Chemistry 280 (33): 29971-79.

https://doi.org/10.1074/jbc.M501931200.

Ge, Wen-Song, Yao-Jun Wang, Jian-Xin Wu, Jian-Gao Fan, Ying-Wei Chen, et Liang Zhu. 2014. « $\beta$ Catenin Is Overexpressed in Hepatic Fibrosis and Blockage of Wnt/ $\beta$-Catenin Signaling Inhibits Hepatic Stellate Cell Activation ». Molecular Medicine Reports 9 (6): 2145-51.

https://doi.org/10.3892/mmr.2014.2099.

Goodwin, B., S. A. Jones, R. R. Price, M. A. Watson, D. D. McKee, L. B. Moore, C. Galardi, et al. 2000. " A Regulatory Cascade of the Nuclear Receptors FXR, SHP-1, and LRH-1 Represses Bile Acid Biosynthesis ». Molecular Cell 6 (3): 517-26. https://doi.org/10.1016/s1097-2765(00)00051-4.

Hartmann, Daniela, Jessica Lucks, Sina Fuchs, Susanne Schiffmann, Yannick Schreiber, Nerea Ferreirós, Jennifer Merkens, Rolf Marschalek, Gerd Geisslinger, et Sabine Grösch. 2012. « Long Chain Ceramides and Very Long Chain Ceramides Have Opposite Effects on Human Breast and Colon Cancer Cell Growth ». The International Journal of Biochemistry \& Cell Biology 44 (4): 620-28.

https://doi.org/10.1016/j.biocel.2011.12.019.

Holt, Peter R. 1972. " The Roles of Bile Acids During the Process of Normal Fat and Cholesterol Absorption ». Archives of Internal Medicine 130 (4): 574-83.

https://doi.org/10.1001/archinte.1972.03650040100009.

Hoogerland, Joanne A., Yu Lei, Justina C. Wolters, Jan Freark de Boer, Trijnie Bos, Aycha Bleeker, Niels L. Mulder, et al. 2019. " Glucose-6-Phosphate Regulates Hepatic Bile Acid Synthesis in Mice ». Hepatology (Baltimore, Md.) 70 (6): 2171-84. https://doi.org/10.1002/hep.30778. 
Hornemann, Thorsten, Yu Wei, et Arnold von Eckardstein. 2007. «Is the mammalian serine palmitoyltransferase a high-molecular-mass complex? » Biochemical Journal 405 (Pt 1): 157-64. https://doi.org/10.1042/BJ20070025.

Insausti-Urkia, Naroa, Estel Solsona-Vilarrasa, Carmen Garcia-Ruiz, et Jose C. Fernandez-Checa. 2020. « Sphingomyelinases and Liver Diseases ». Biomolecules 10 (11): E1497.

https://doi.org/10.3390/biom10111497.

Jiang, Changtao, Cen Xie, Ying Lv, Jing Li, Kristopher W. Krausz, Jingmin Shi, Chad N. Brocker, et al. 2015. « Intestine-Selective Farnesoid X Receptor Inhibition Improves Obesity-Related Metabolic Dysfunction ». Nature Communications 6 (décembre): 10166.

https://doi.org/10.1038/ncomms10166.

Jiang, Meng, Chun Li, Qiaoshu Liu, Aimin Wang, et Minxiang Lei. 2019. «Inhibiting Ceramide Synthesis Attenuates Hepatic Steatosis and Fibrosis in Rats With Non-Alcoholic Fatty Liver Disease ». Frontiers in Endocrinology 10. https://doi.org/10.3389/fendo.2019.00665.

Kasumov, Takhar, Ling Li, Min Li, Kailash Gulshan, John P. Kirwan, Xiuli Liu, Stephen Previs, Belinda Willard, Jonathan D. Smith, et Arthur McCullough. 2015. "Ceramide as a Mediator of Non-Alcoholic Fatty Liver Disease and Associated Atherosclerosis ». PLOS ONE 10 (5): e0126910. https://doi.org/10.1371/journal.pone.0126910.

Kitatani, Kazuyuki, Jolanta Idkowiak-Baldys, et Yusuf A. Hannun. 2008. « The sphingolipid salvage pathway in ceramide metabolism and signaling ». Cellular signalling 20 (6): 1010-18. https://doi.org/10.1016/j.cellsig.2007.12.006.

Li, Zhiqiang, Inamul Kabir, Hui Jiang, Hongwen Zhou, Jenny Libien, Jianying Zeng, Albert Stanek, et al. 2016. « Liver Serine Palmitoyltransferase (SPT) Activity Deficiency in Early Life Impairs Adherens Junctions and Promotes Tumorigenesis ». Hepatology (Baltimore, Md.) 64 (6): 2089-2102. https://doi.org/10.1002/hep.28845.

Mithieux, Gilles, Isabelle Bady, Amandine Gautier, Martine Croset, Fabienne Rajas, et Carine Zitoun. 2004. «Induction of Control Genes in Intestinal Gluconeogenesis Is Sequential during Fasting and Maximal in Diabetes ». American Journal of Physiology. Endocrinology and Metabolism 286 (3): E370375. https://doi.org/10.1152/ajpendo.00299.2003.

Péan, Noémie, Isabelle Doignon, Isabelle Garcin, Aurore Besnard, Boris Julien, Bingkaï Liu, Sophie Branchereau, et al. 2013. "The Receptor TGR5 Protects the Liver from Bile Acid Overload during Liver Regeneration in Mice ». Hepatology 58 (4): 1451-60. https://doi.org/10.1002/hep.26463.

Penhoat, Armelle, Laetitia Fayard, Anne Stefanutti, Gilles Mithieux, et Fabienne Rajas. 2014. « Intestinal Gluconeogenesis Is Crucial to Maintain a Physiological Fasting Glycemia in the Absence of Hepatic Glucose Production in Mice ». Metabolism: Clinical and Experimental 63 (1): 104-11. https://doi.org/10.1016/j.metabol.2013.09.005.

Pewzner-Jung, Yael, Ori Brenner, Svantje Braun, Elad L. Laviad, Shifra Ben-Dor, Ester Feldmesser, Shirley Horn-Saban, et al. 2010. « A Critical Role for Ceramide Synthase 2 in Liver Homeostasis: II. Insights into Molecular Changes Leading to Hepatopathy ». The Journal of Biological Chemistry 285 (14): 10911-23. https://doi.org/10.1074/jbc.M109.077610.

Poitout, Vincent, et R. Paul Robertson. 2008. « Glucolipotoxicity: Fuel Excess and Beta-Cell Dysfunction ». Endocrine Reviews 29 (3): 351-66. https://doi.org/10.1210/er.2007-0023. 
Raichur, Suryaprakash, Bodo Brunner, Maximilian Bielohuby, Gitte Hansen, Anja Pfenninger, Bing Wang, Jens C. Bruning, Philip Just Larsen, et Norbert Tennagels. 2019. « The Role of C16:0 Ceramide in the Development of Obesity and Type 2 Diabetes: CerS6 Inhibition as a Novel Therapeutic Approach ». Molecular Metabolism 21 (mars): 36-50. https://doi.org/10.1016/j.molmet.2018.12.008.

Raichur, Suryaprakash, Siew Tein Wang, Puck Wee Chan, Ying Li, Jianhong Ching, Bhagirath Chaurasia, Bghagirath Chaurasia, et al. 2014. "CerS2 Haploinsufficiency Inhibits $\beta$-Oxidation and Confers Susceptibility to Diet-Induced Steatohepatitis and Insulin Resistance ». Cell Metabolism 20 (4): 687-95. https://doi.org/10.1016/j.cmet.2014.09.015.

Rajas, F., N. Bruni, S. Montano, C. Zitoun, et G. Mithieux. 1999. "The Glucose-6 Phosphatase Gene Is Expressed in Human and Rat Small Intestine: Regulation of Expression in Fasted and Diabetic Rats ». Gastroenterology 117 (1): 132-39. https://doi.org/10.1016/s0016-5085(99)70559-7.

Rodriguez-Cuenca, S., N. Barbarroja, et A. Vidal-Puig. 2015. « Dihydroceramide Desaturase 1, the Gatekeeper of Ceramide Induced Lipotoxicity ». Biochimica Et Biophysica Acta 1851 (1): 40-50. https://doi.org/10.1016/j.bbalip.2014.09.021.

Roehrig, K. L., et J. B. Allred. 1974. « Direct Enzymatic Procedure for the Determination of Liver Glycogen ». Analytical Biochemistry 58 (2): 414-21. https://doi.org/10.1016/0003-2697(74)90210-3.

Sayin, Sama I., Annika Wahlström, Jenny Felin, Sirkku Jäntti, Hanns-Ulrich Marschall, Krister Bamberg, Bo Angelin, Tuulia Hyötyläinen, Matej Orešič, et Fredrik Bäckhed. 2013. " Gut Microbiota Regulates Bile Acid Metabolism by Reducing the Levels of Tauro-Beta-Muricholic Acid, a Naturally Occurring FXR Antagonist ». Cell Metabolism 17 (2): 225-35. https://doi.org/10.1016/j.cmet.2013.01.003.

Siddique, Monowarul Mobin, Ying Li, Bhagirath Chaurasia, Vincent A. Kaddai, et Scott A. Summers. 2015. " Dihydroceramides: From Bit Players to Lead Actors ». The Journal of Biological Chemistry 290 (25): 15371-79. https://doi.org/10.1074/jbc.R115.653204.

Si-Tayeb, Karim, Frédéric P. Lemaigre, et Stephen A. Duncan. 2010. « Organogenesis and Development of the Liver ». Developmental Cell 18 (2): 175-89.

https://doi.org/10.1016/j.devcel.2010.01.011.

Stayrook, Keith R., Kelli S. Bramlett, Rajesh S. Savkur, James Ficorilli, Todd Cook, Michael E. Christe, Laura F. Michael, et Thomas P. Burris. 2005. « Regulation of Carbohydrate Metabolism by the Farnesoid X Receptor ». Endocrinology 146 (3): 984-91. https://doi.org/10.1210/en.2004-0965.

Sun, Lulu, Jie Cai, et Frank J. Gonzalez. 2021. « The Role of Farnesoid X Receptor in Metabolic Diseases, and Gastrointestinal and Liver Cancer ». Nature Reviews. Gastroenterology \& Hepatology 18 (5): 335-47. https://doi.org/10.1038/s41575-020-00404-2.

Viollet, Benoit, Fabrizio Andreelli, Sebastian B. Jørgensen, Christophe Perrin, Alain Geloen, Daisy Flamez, James Mu, et al. 2003. "The AMP-Activated Protein Kinase Alpha2 Catalytic Subunit Controls Whole-Body Insulin Sensitivity ». The Journal of Clinical Investigation 111 (1): 91-98. https://doi.org/10.1172/JCl16567.

Wigger, Leonore, Céline Cruciani-Guglielmacci, Anthony Nicolas, Jessica Denom, Neïké Fernandez, Frédéric Fumeron, Pedro Marques-Vidal, et al. 2017. «Plasma Dihydroceramides Are Diabetes Susceptibility Biomarker Candidates in Mice and Humans ». Cell Reports 18 (9): 2269-79. https://doi.org/10.1016/j.celrep.2017.02.019. 
Wu, Qing, Lulu Sun, Xiaomin Hu, Xuemei Wang, Feng Xu, Bo Chen, Xianyi Liang, et al. 2021. "Suppressing the Intestinal Farnesoid X Receptor/Sphingomyelin Phosphodiesterase 3 Axis Decreases Atherosclerosis ». The Journal of Clinical Investigation 131 (9).

https://doi.org/10.1172/JCl142865.

Xie, Cen, Changtao Jiang, Jingmin Shi, Xiaoxia Gao, Dongxue Sun, Lulu Sun, Ting Wang, et al. 2017. " An Intestinal Farnesoid X Receptor-Ceramide Signaling Axis Modulates Hepatic Gluconeogenesis in Mice ». Diabetes 66 (3): 613-26. https://doi.org/10.2337/db16-0663.

Zollner, G., P. Fickert, R. Zenz, A. Fuchsbichler, C. Stumptner, L. Kenner, P. Ferenci, et al. 2001. " Hepatobiliary Transporter Expression in Percutaneous Liver Biopsies of Patients with Cholestatic Liver Diseases ». Hepatology (Baltimore, Md.) 33 (3): 633-46.

https://doi.org/10.1053/jhep.2001.22646. 


\section{Figure 1.}

A.
Spt/c2 ${ }^{\text {WTHep }} \mathrm{RC}$
Spt/c2 $2^{\Delta \mathrm{Hep}} \mathrm{RC}$

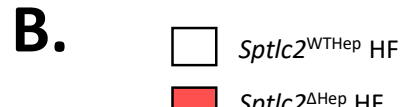

Sptlc2 $2^{\text {AHep }} \mathrm{HF}$

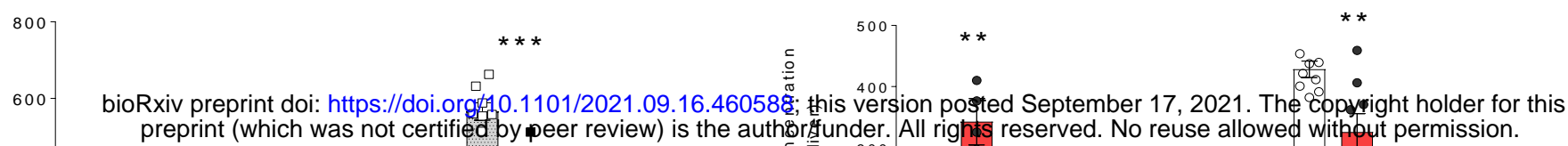

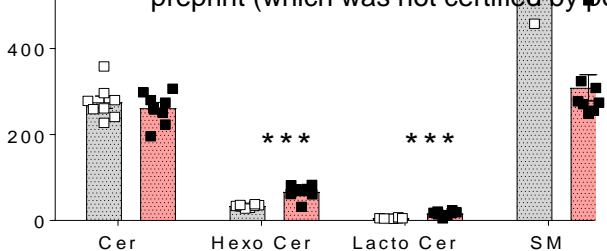

C.
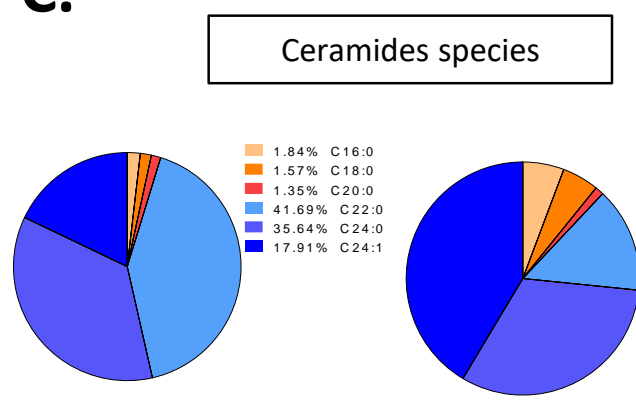

= $5.72 \%$ C $16: 0$

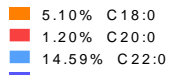

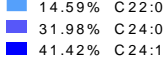

Spt/c2 ${ }^{\text {WTHep RC }}$

Sptlc2 $2^{\Delta \mathrm{Hep}} \mathrm{RC}$

E.

B. Spt/c2 ${ }^{\text {WTHep }} \mathrm{RC}$

Spt/c2 ${ }^{\Delta \text { Hep } R C}$

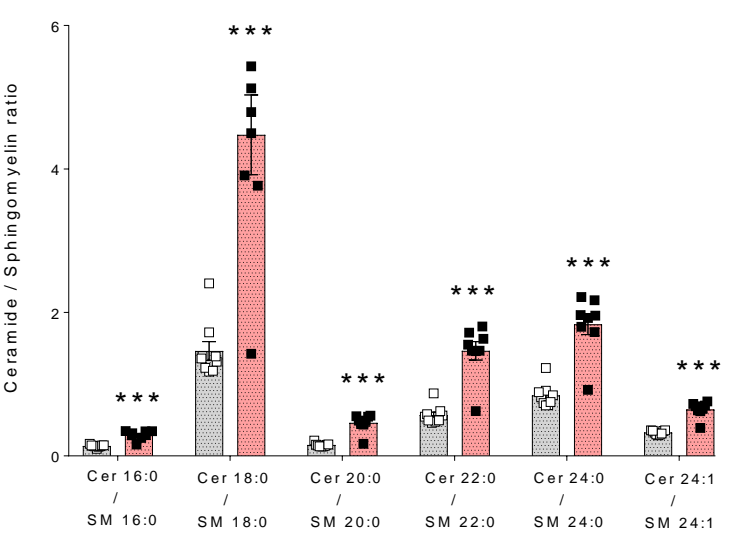

G.

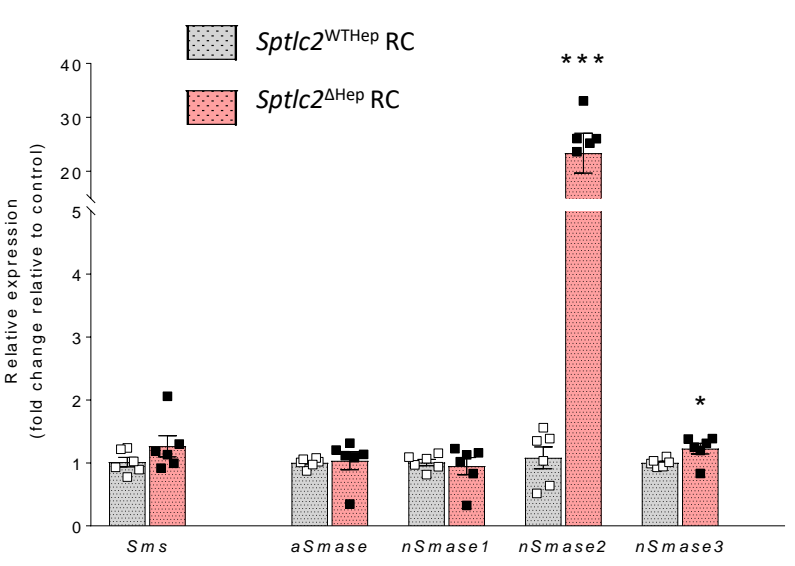

I.

nSmase2 (71 kDa)

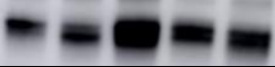

CYCLO-a (18 kDa)

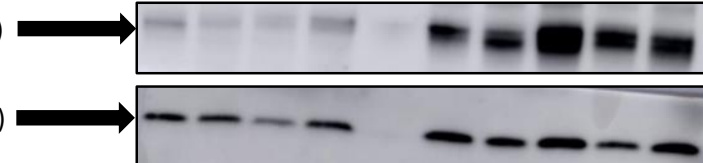

D.

Ceramides species
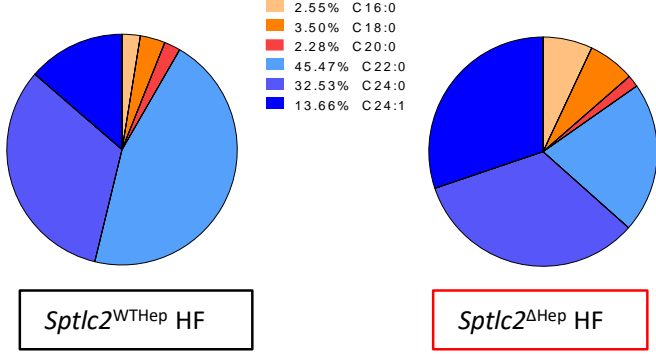

$7.00 \%$ C $16: 0$
$6.62 \%$
C 18.0 $1.68 \%$ c $20: 0$
$21.26 \% \quad c 22: 0$ E $33.26 \%$ c $24: 0$

F.

Sptlc $2^{\text {WTHep }} \mathrm{HF}$

Sptlc2 $2^{\text {मHep }} \mathrm{HF}$

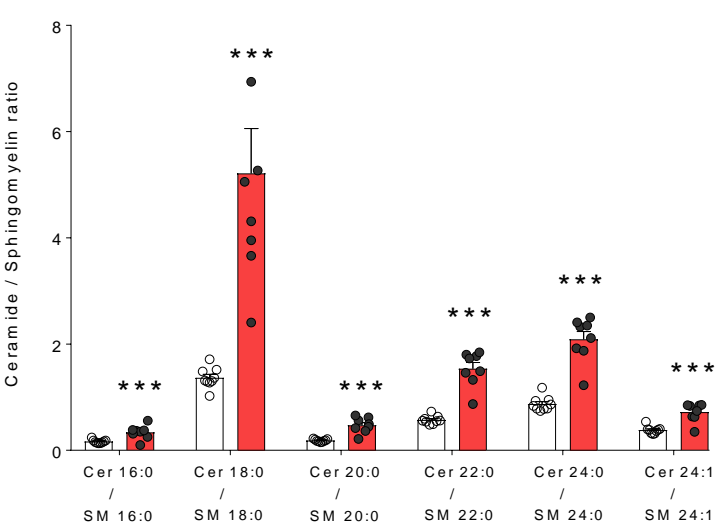

H.

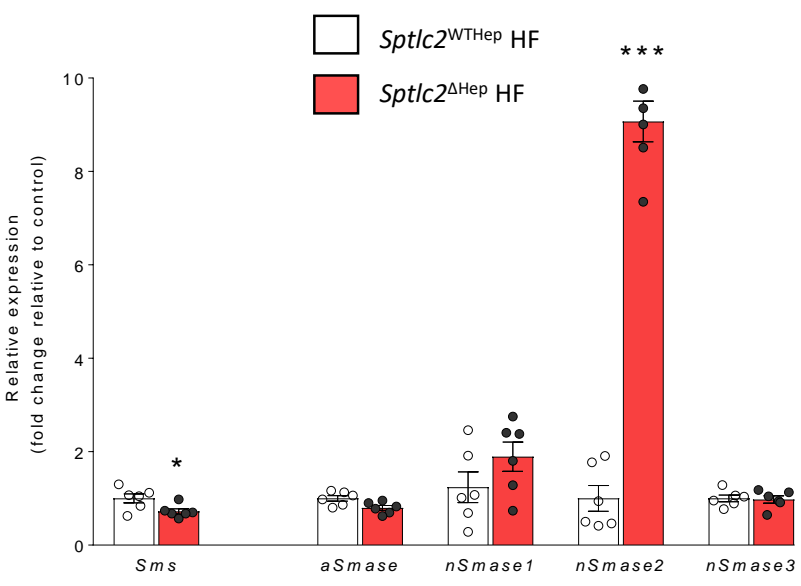

. Sptlc2 ${ }^{\text {WTHep }} \mathrm{RC}$

Spt/c2 $2^{\Delta \mathrm{Hep}} \mathrm{RC}$

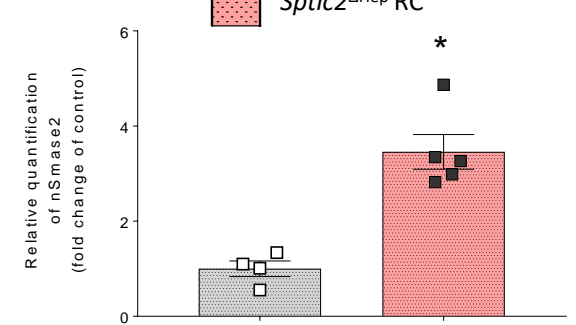




\section{Figure 2.}

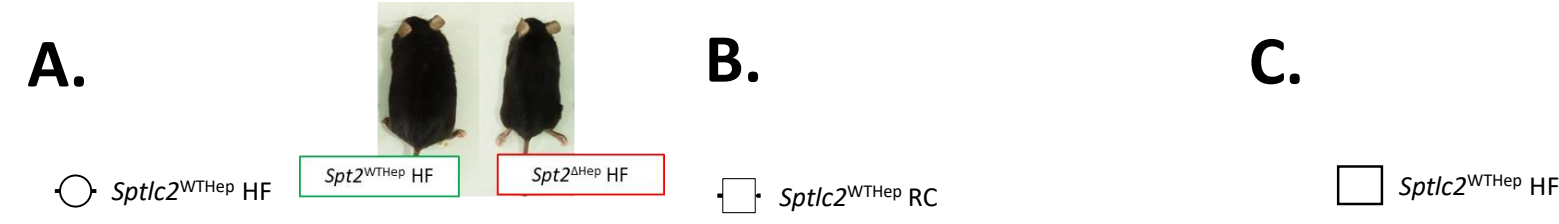

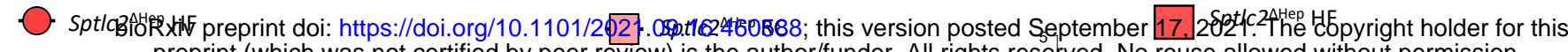
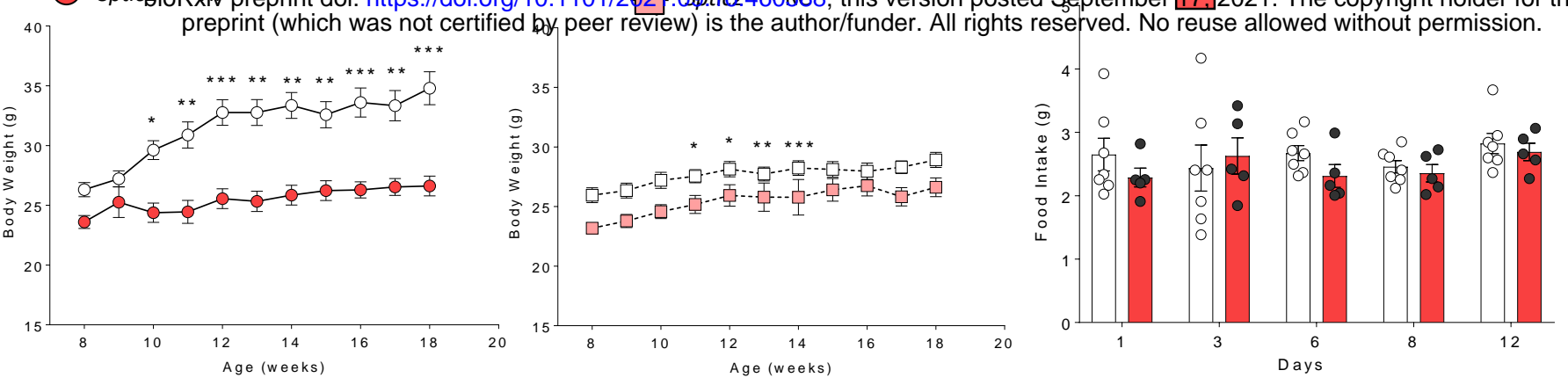

D.

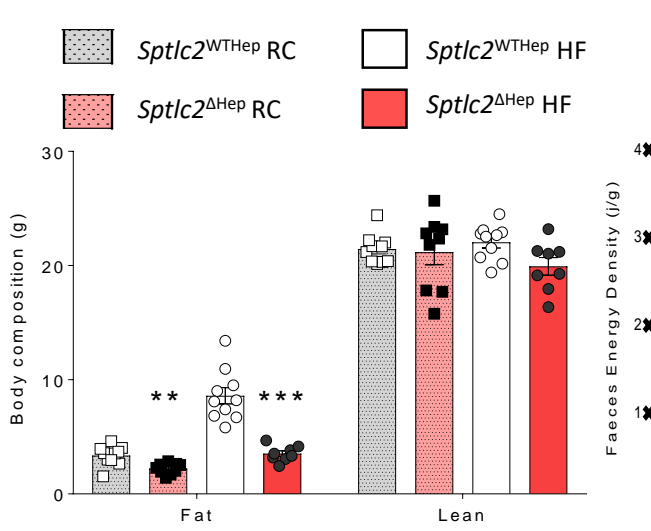

G
I.
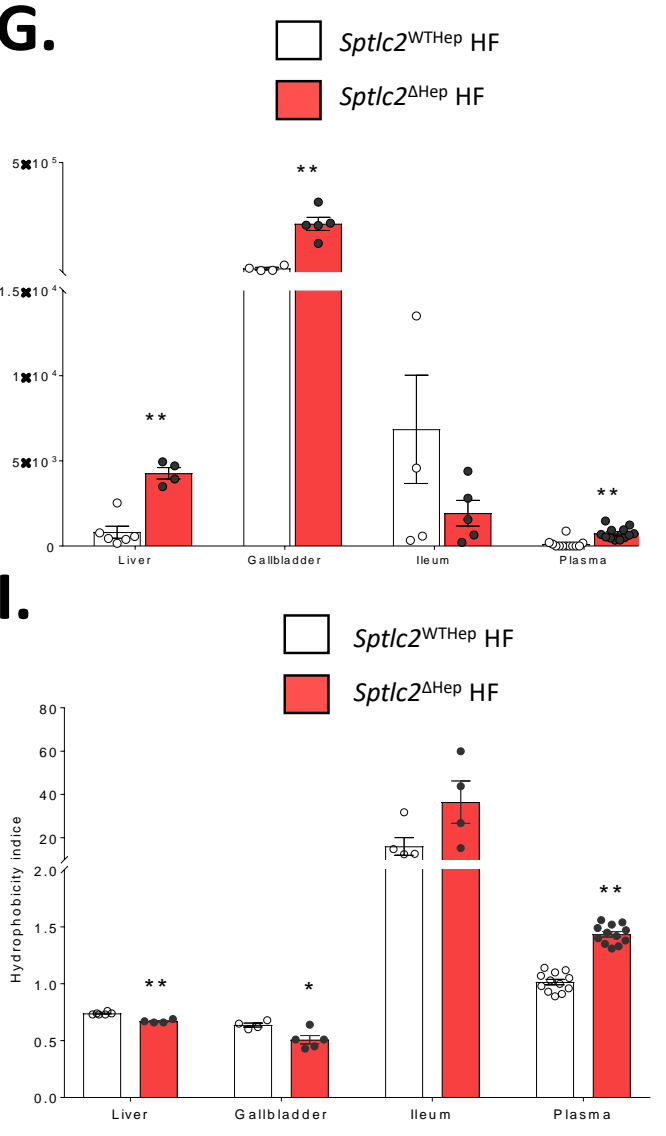

E.

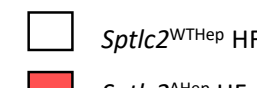

Sptlc2 $2^{\text {Hep }} \mathrm{HF}$

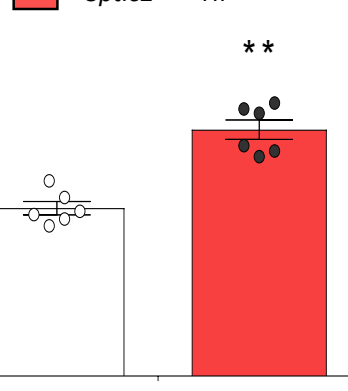

F.

H.
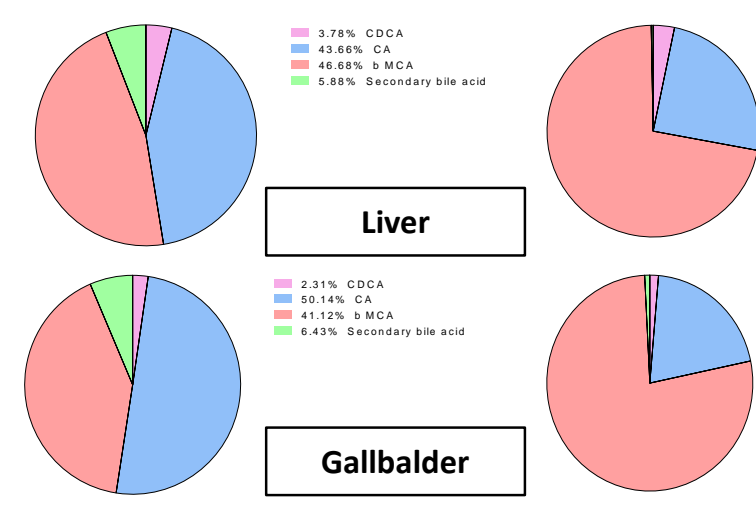

$=2.24 \% \mathrm{CDCA}$

- Spt/c2 ${ }^{\text {WTHep }} \mathrm{HF}$

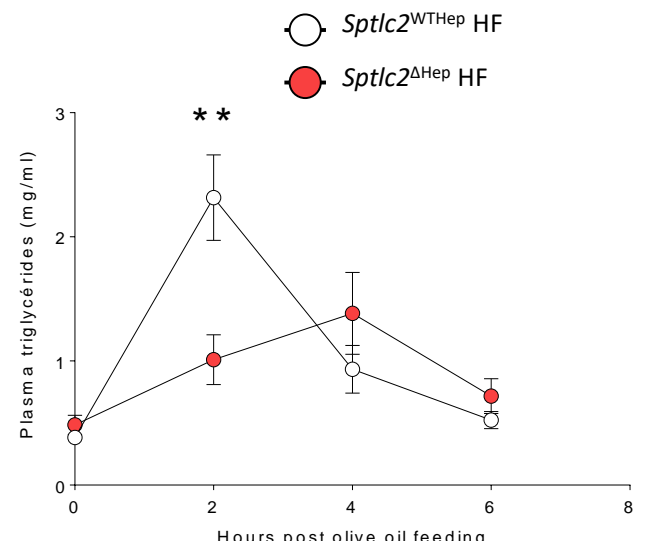

J.

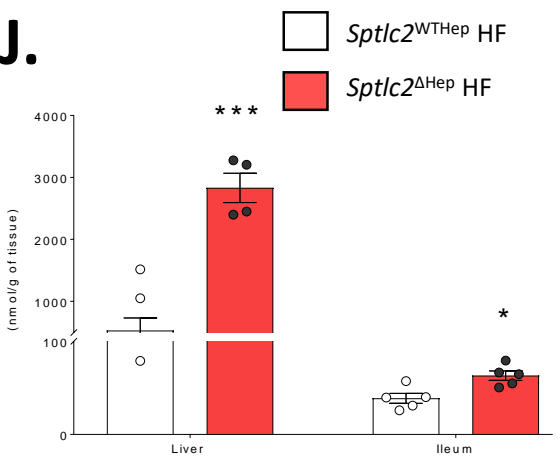

K.
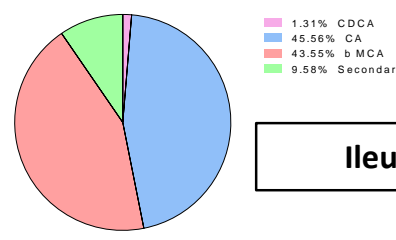

Ileum

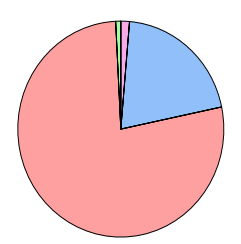

$1.35 \% \mathrm{CDCA}$
$20.36 \% \mathrm{CA}$

$=2.35 \%$ CA
$20.36 \%$ CA
$77.49 \%$ b MCA

$0.80 \%$ Secondary bile acid.
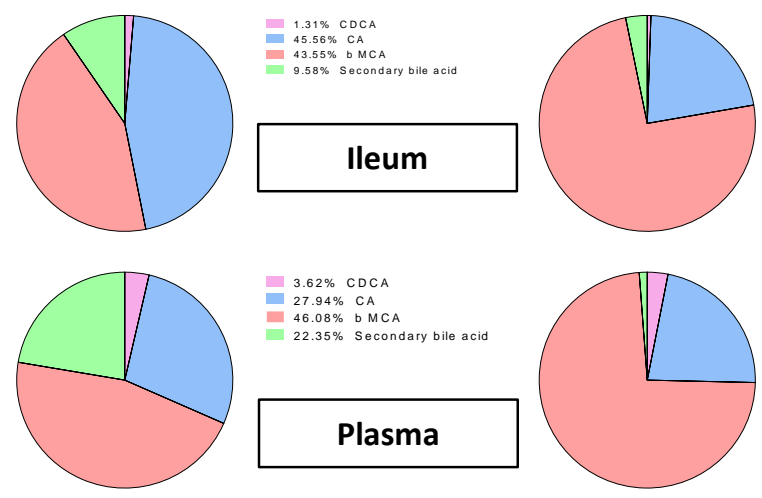

$=0.5 \% \%$ CDCA

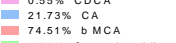

Spt/c2 ${ }^{\text {WTHep HF }}$

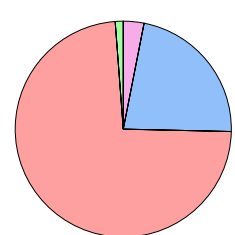

$=\begin{aligned} & 3.09 \% \text { CDCA } \\ & 2.27 \% \text { CA }\end{aligned}$

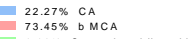

Spt/c2 ${ }^{\text {Hep HF }}$

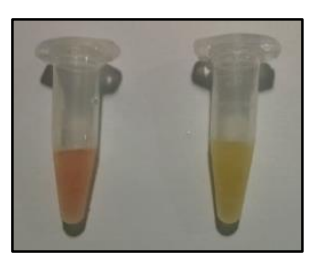


Figure 2.
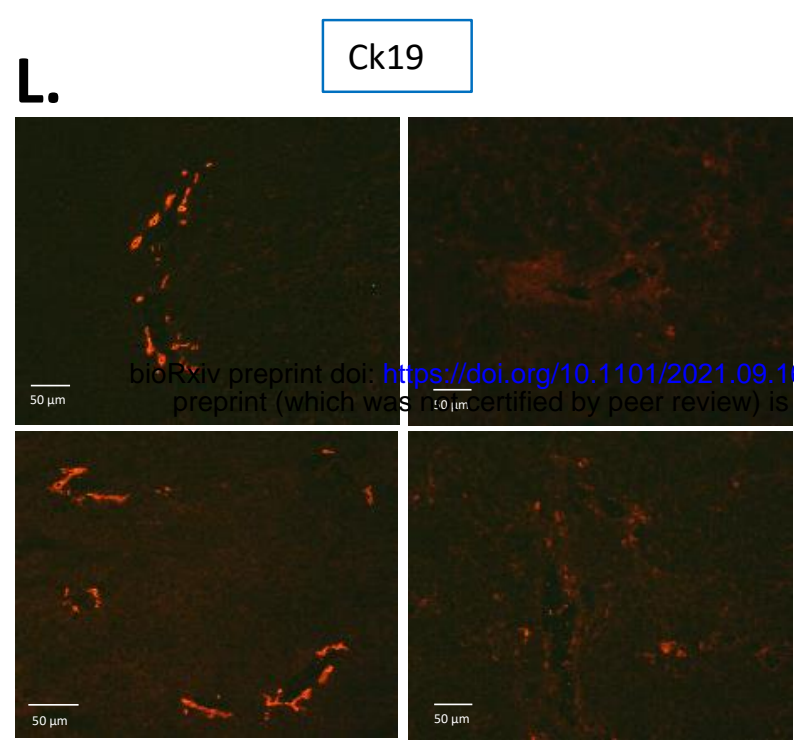

Sptlc2 ${ }^{\text {WTHep }}$ RC

Sptlc2 $^{\mathrm{HHep}} \mathrm{RC}$

6.460588; this version posted September 17, 2021. The copyright holder for this the author/funder. All rights reserved. No reuse allowed without permission.

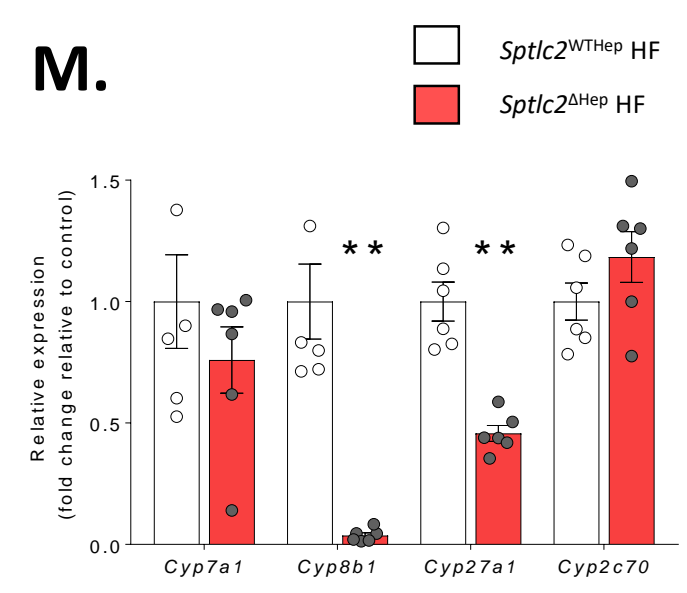

N. Spt/c2 ${ }^{\text {WTHep }} \mathrm{HF}$

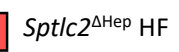
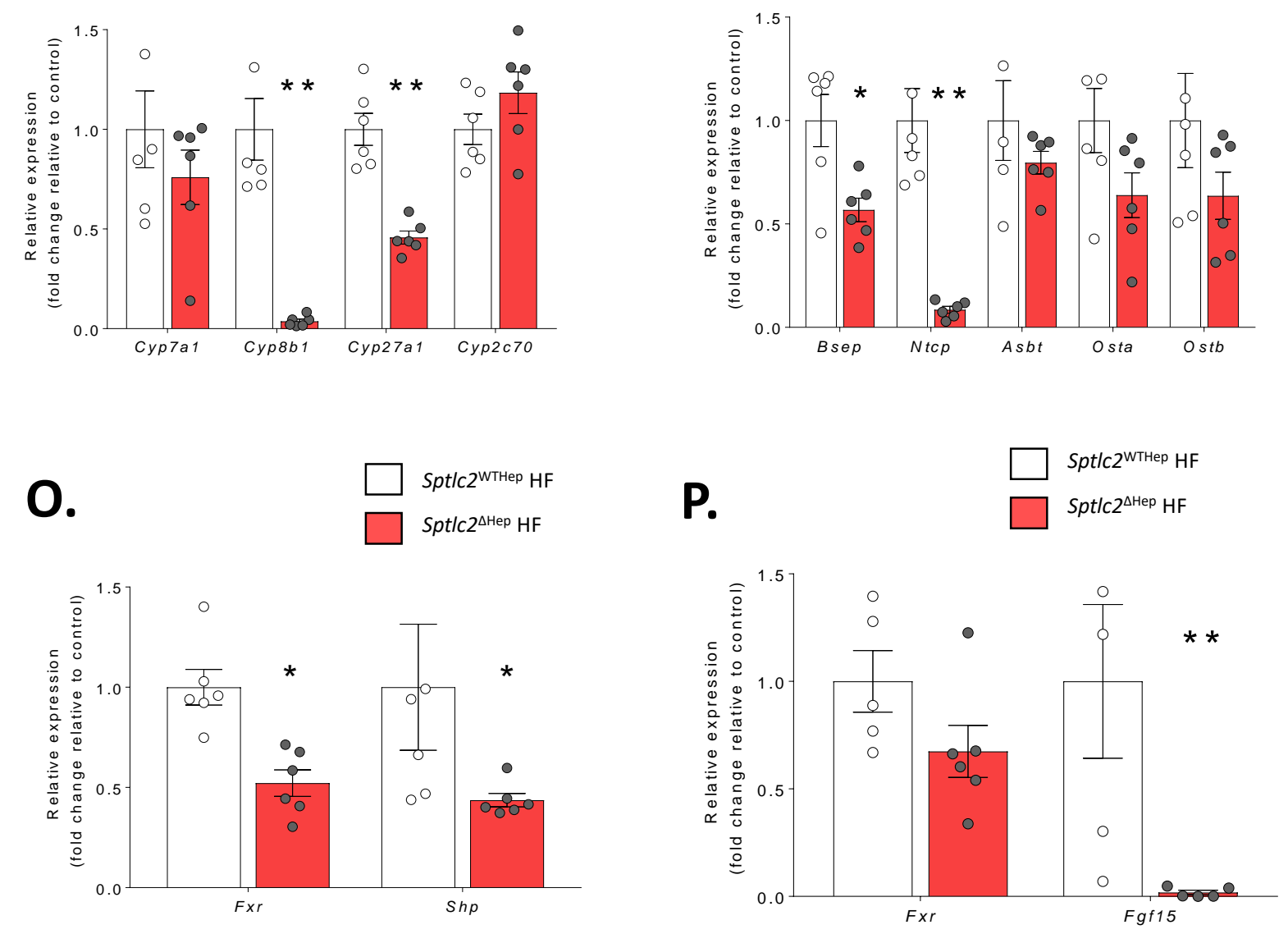
Figure 3.
A.
B. $\bigcirc$ sptlc2 ${ }^{\text {WTHep }} \mathrm{HF}$
- sptlc2 $2^{\text {Hep }} \mathrm{HF}$
C.
Sptlc2 WTHep RC $\square$ sptlc2 ${ }^{\text {WTHep }} \mathrm{HF}$

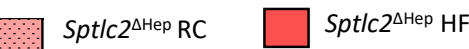
ㄴ. Sptlc2 WTHep RC
‥ Sptlc2 2 Hep RC

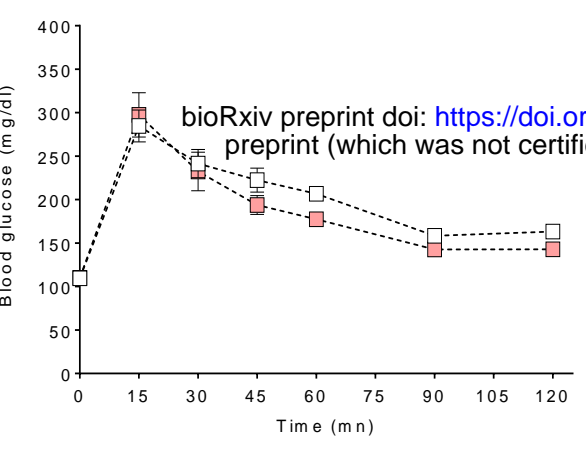

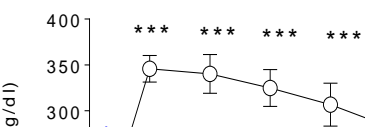
40000

$\star \star *$

$0.1101 / 2021.09 .16 .460588$; this version posted September 17, 2021. The copyright holder for this

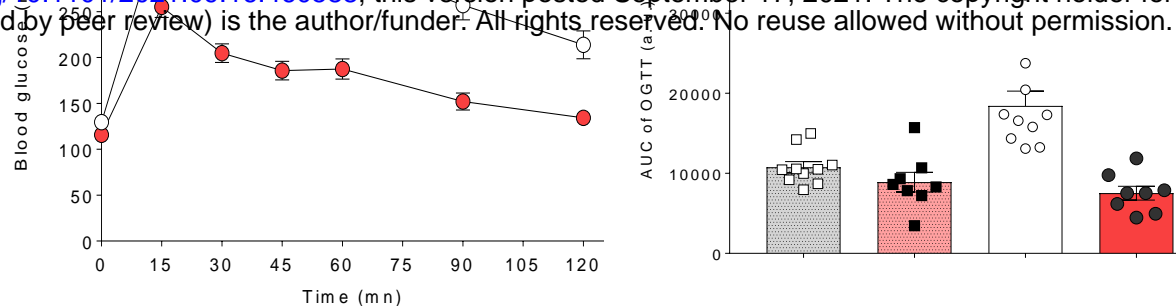
D. $\square$. sptlc2 $^{\text {WTHep }} \mathrm{RC}$

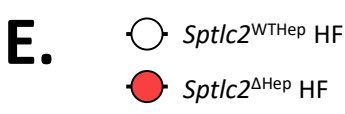

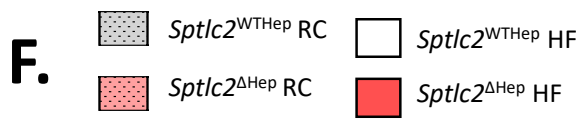
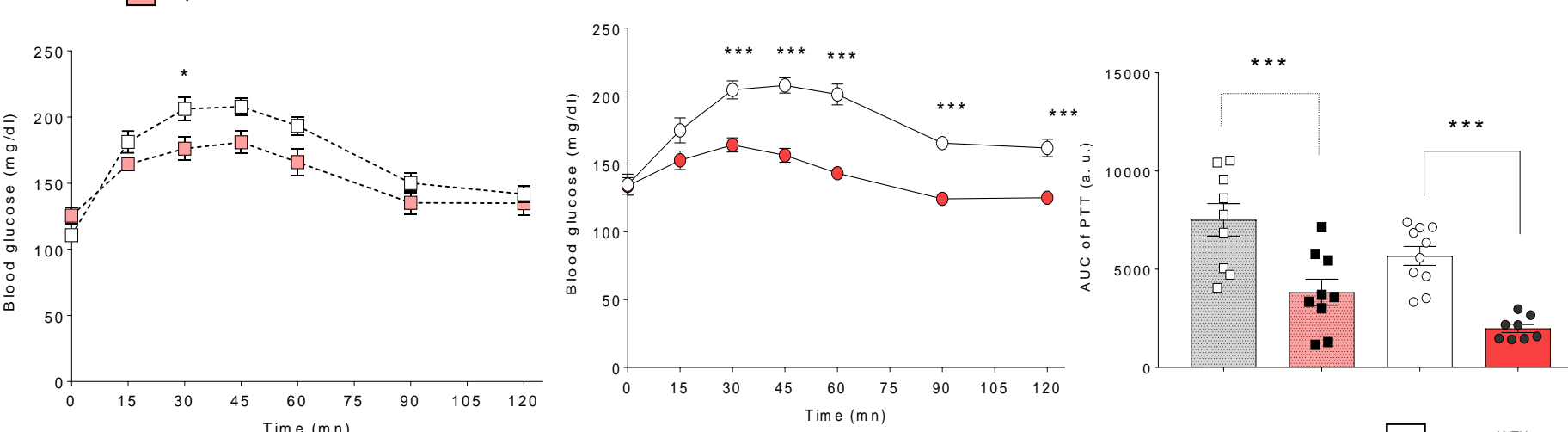

G. $\square \cdot$ spt/c2 $^{\text {WTHep }} \mathrm{RC}$

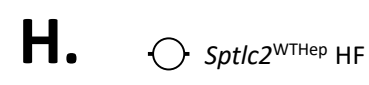

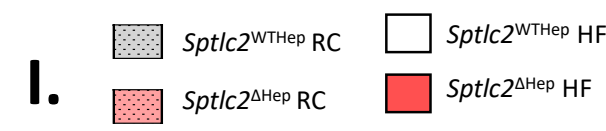
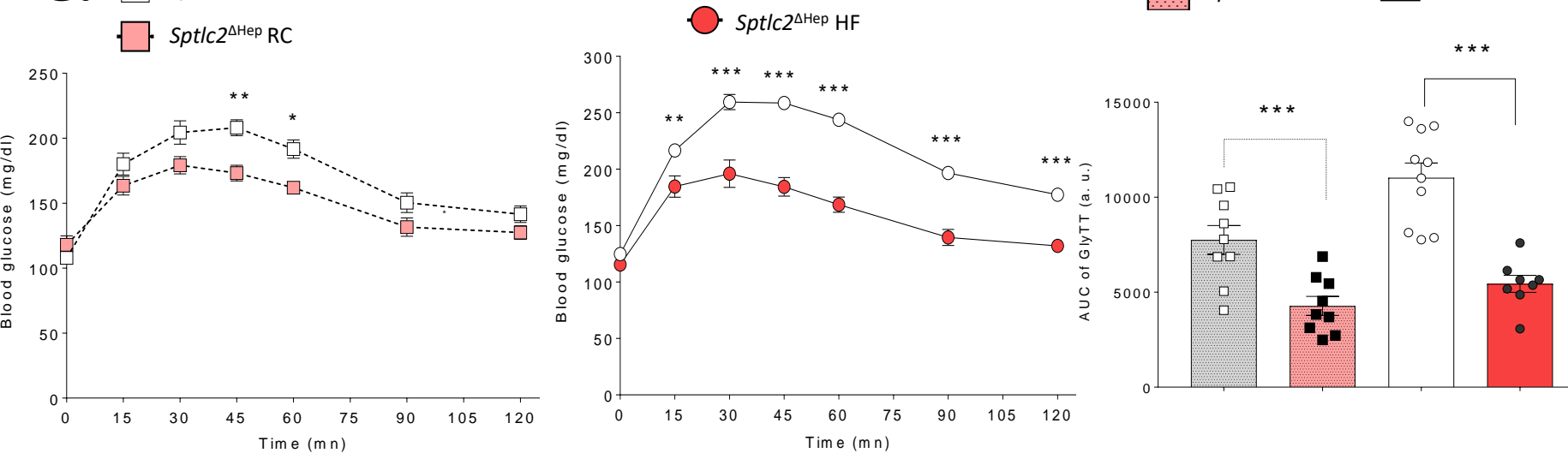

J.
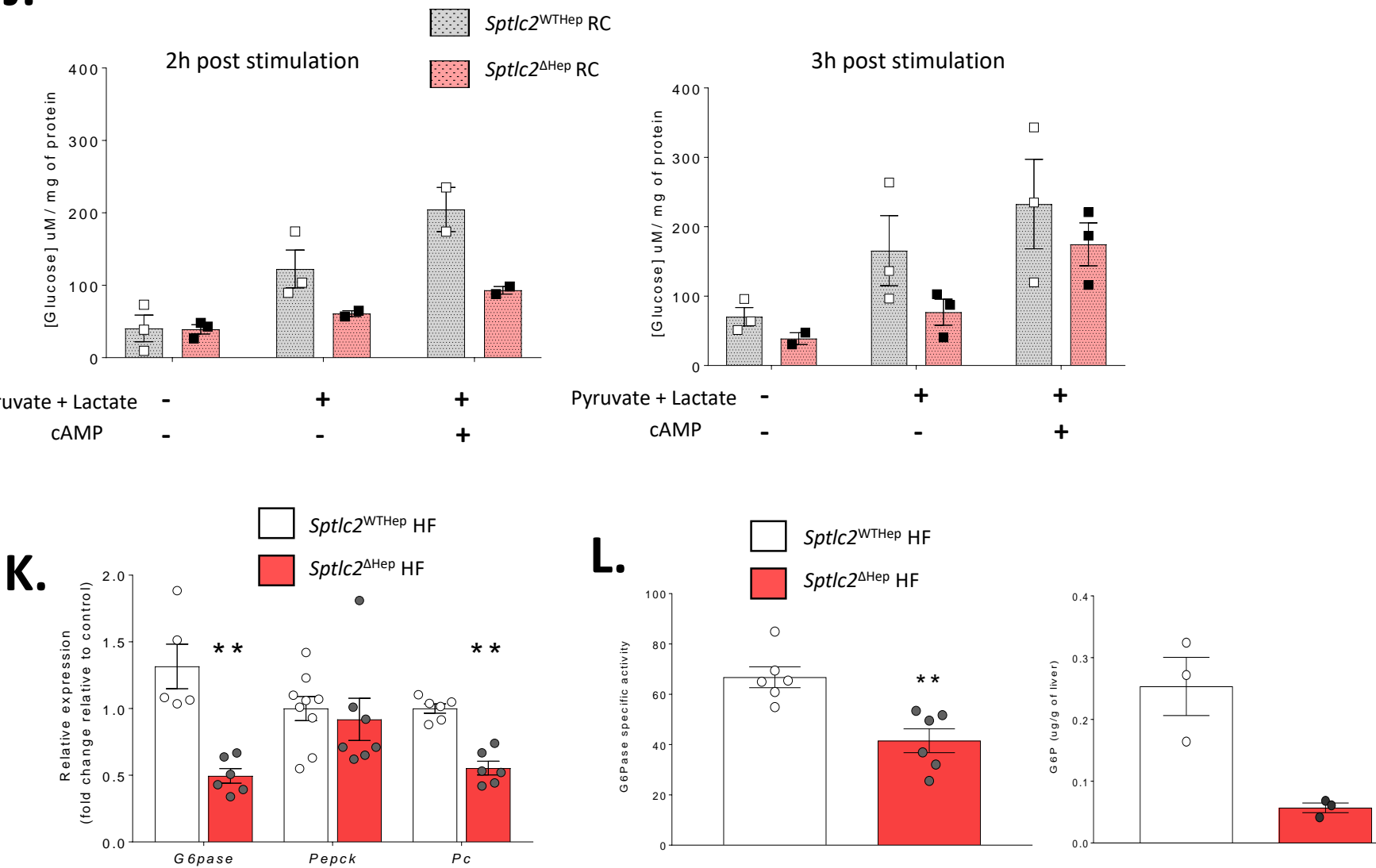

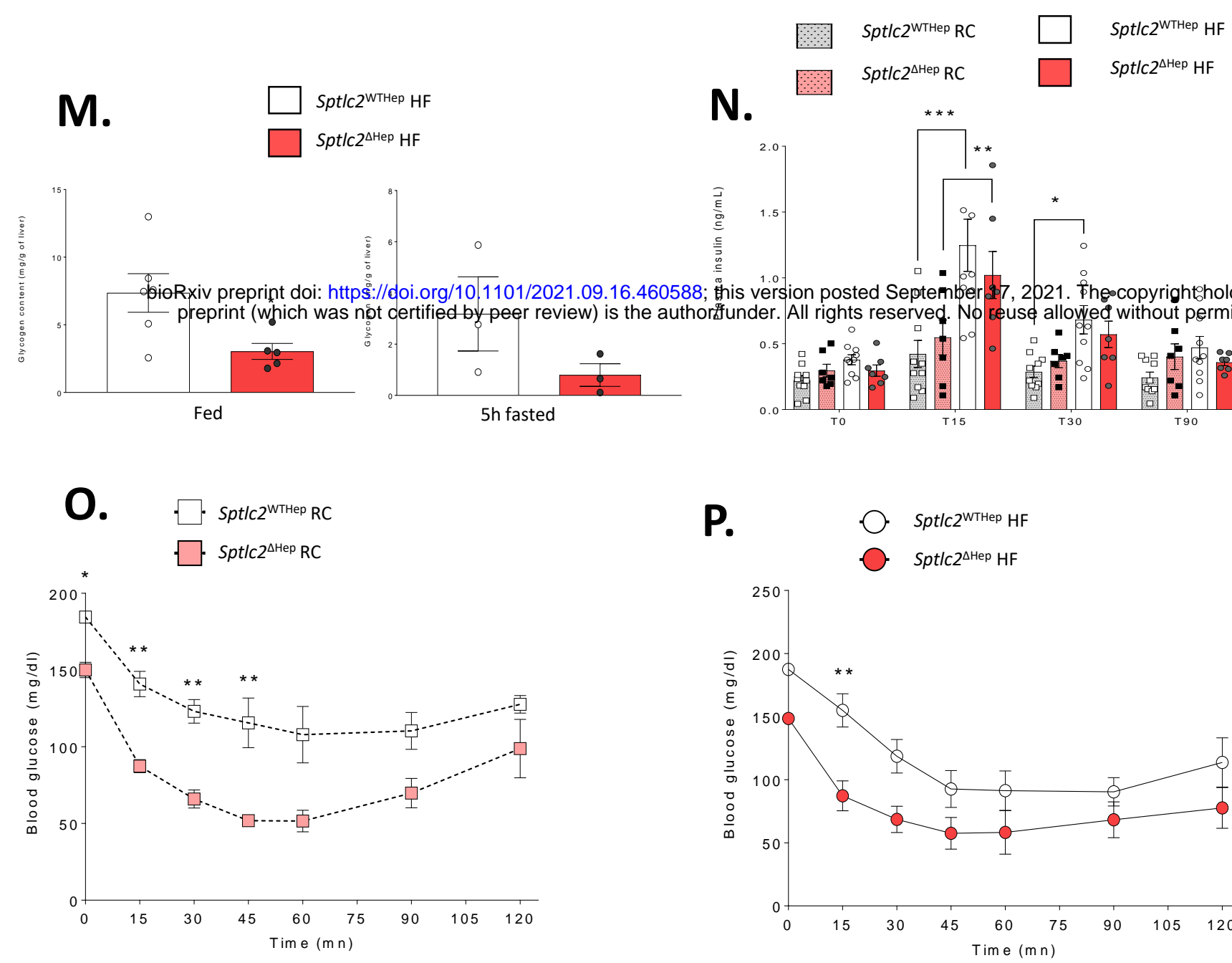

Spt/c2 ${ }^{\text {WTHep }} \mathrm{RC}$

Spt/c2 ${ }^{\mathrm{AHep}} \mathrm{RC}$

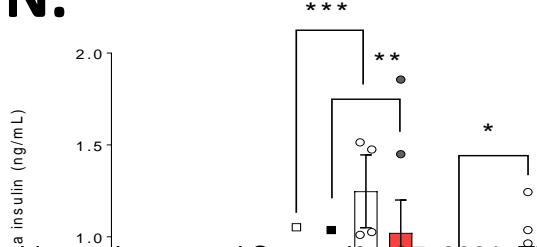

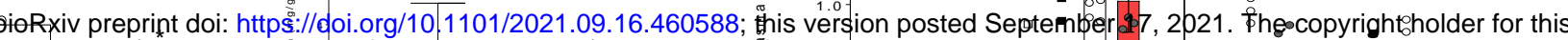
preprint (which was not certified by peer review) is the authorffunder. All rights reserved. No seuse alloweđ̆ without permission.

Fed
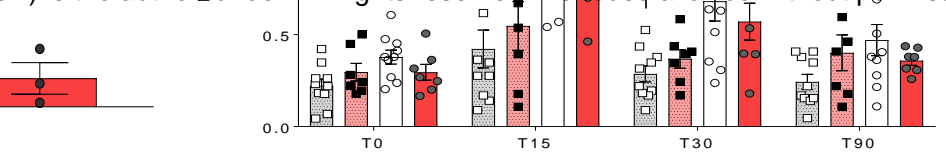

P.

- Spt/c2 ${ }^{\text {WTHep }} \mathrm{HF}$
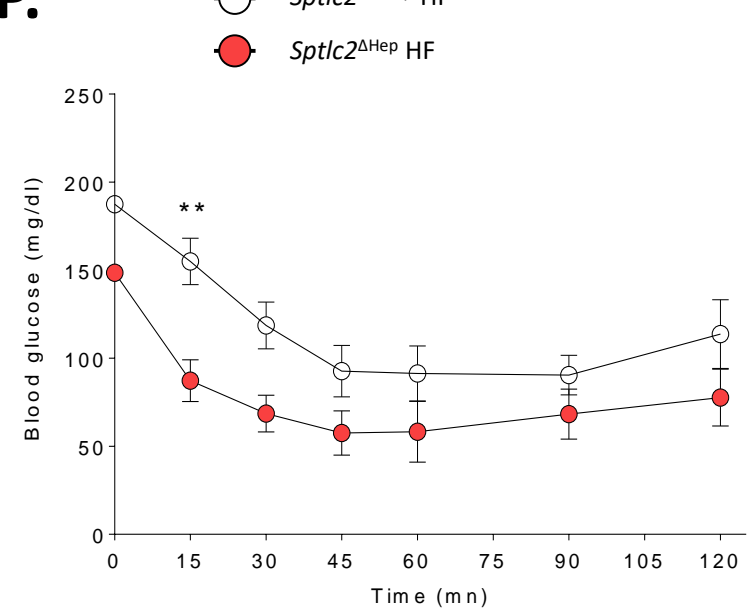

$\square$ Spt/c2 ${ }^{\text {WTHep }}$ HF

Spt/c2 $2^{\Delta \mathrm{Hep}} \mathrm{HF}$
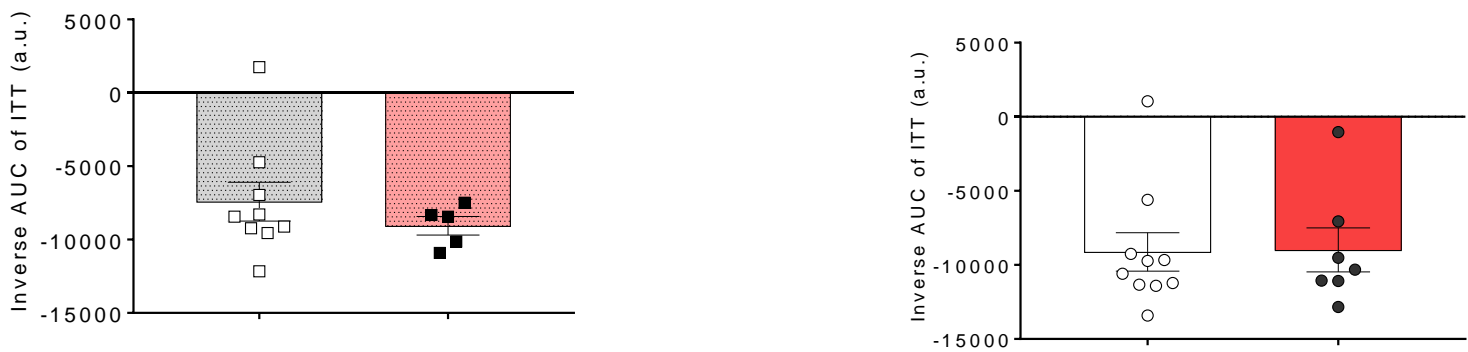

Q.
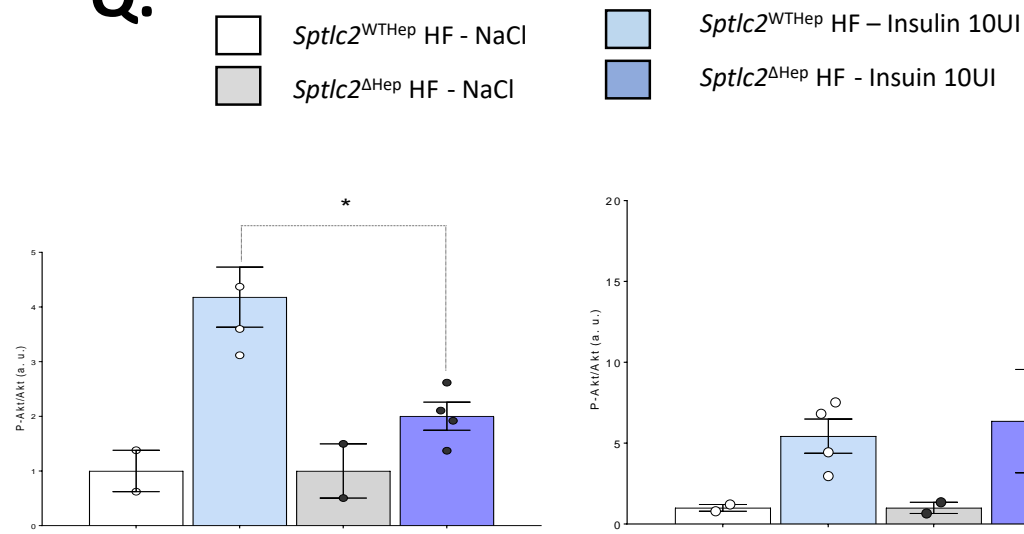

Liver

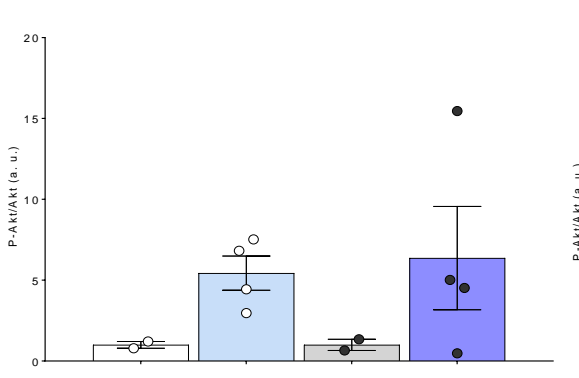

Adipose Tissue

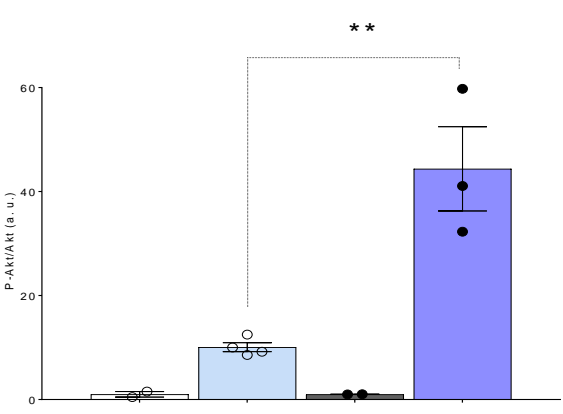

Muscle 
Figure 4.

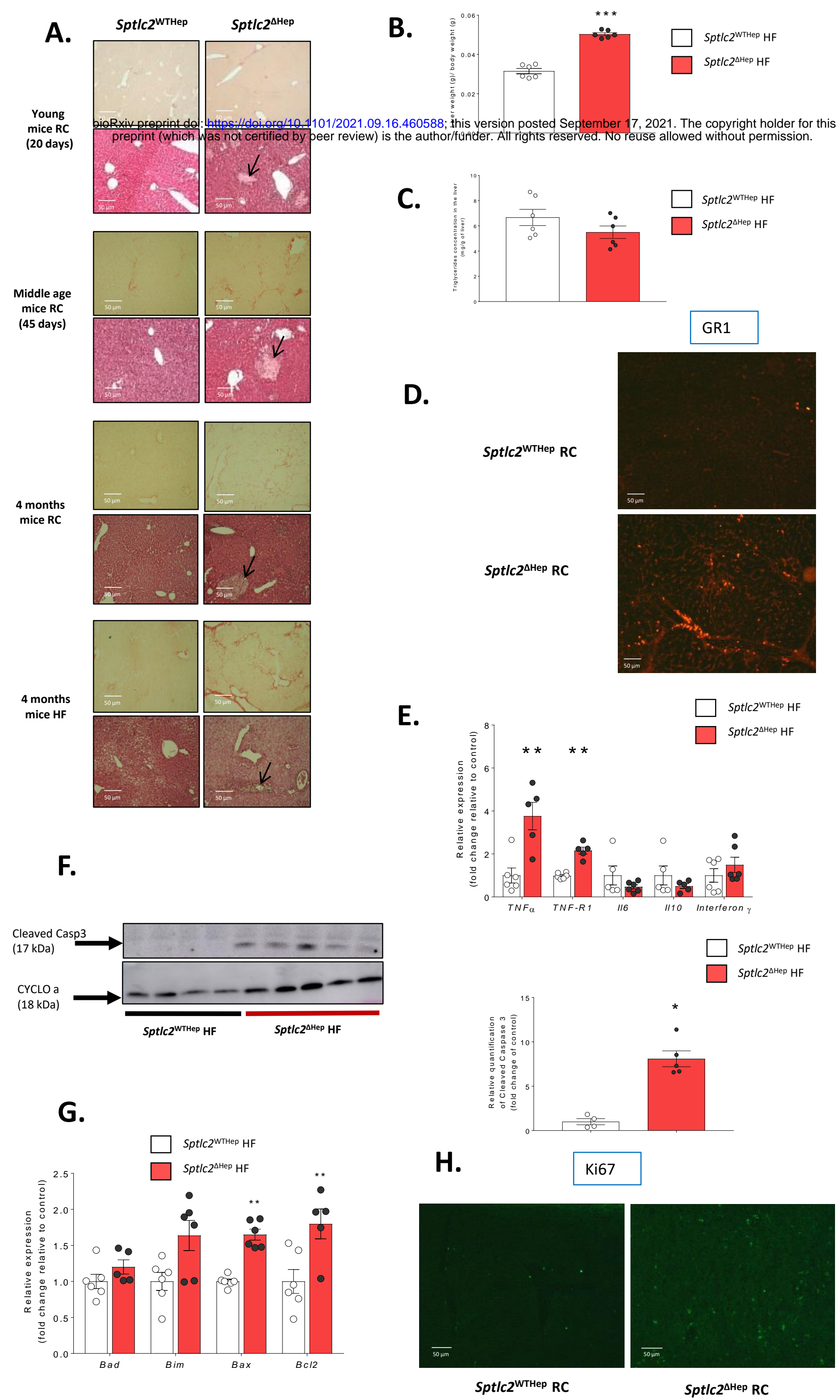




\section{Figure S1.}

A.

B.

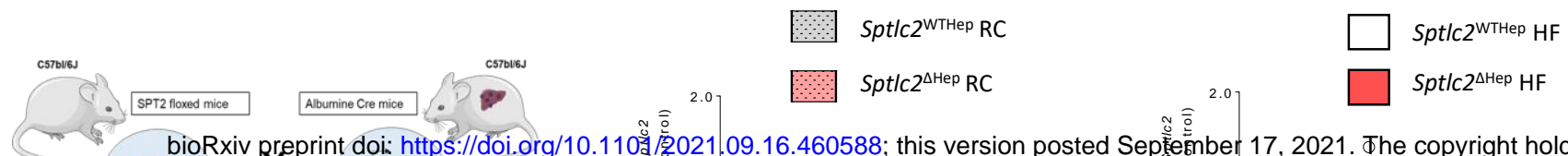

bioRxiv preprint doi: https://doi.org/10.110: + prepryt (whieh was-not certified by peeger deview) is the author/funder. All rights reserved. No reuse allowed without permission.
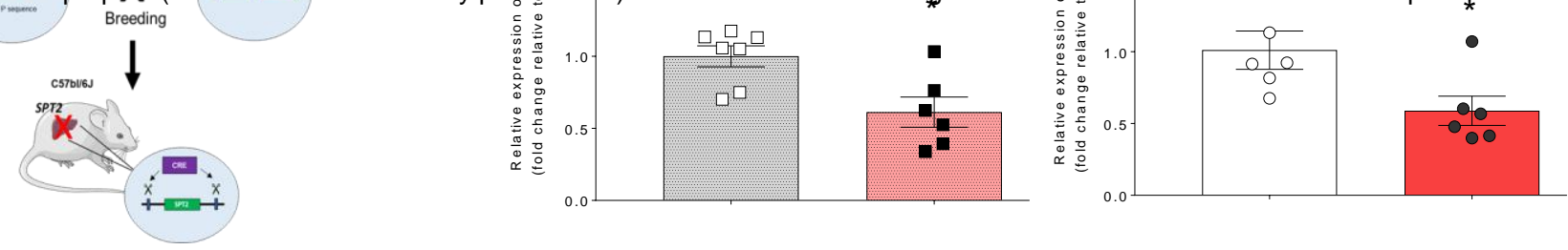

C.

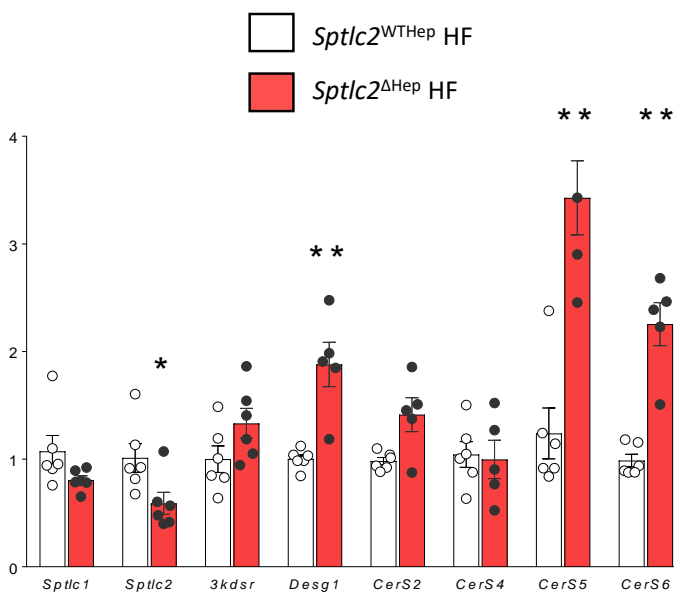

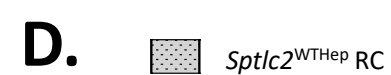
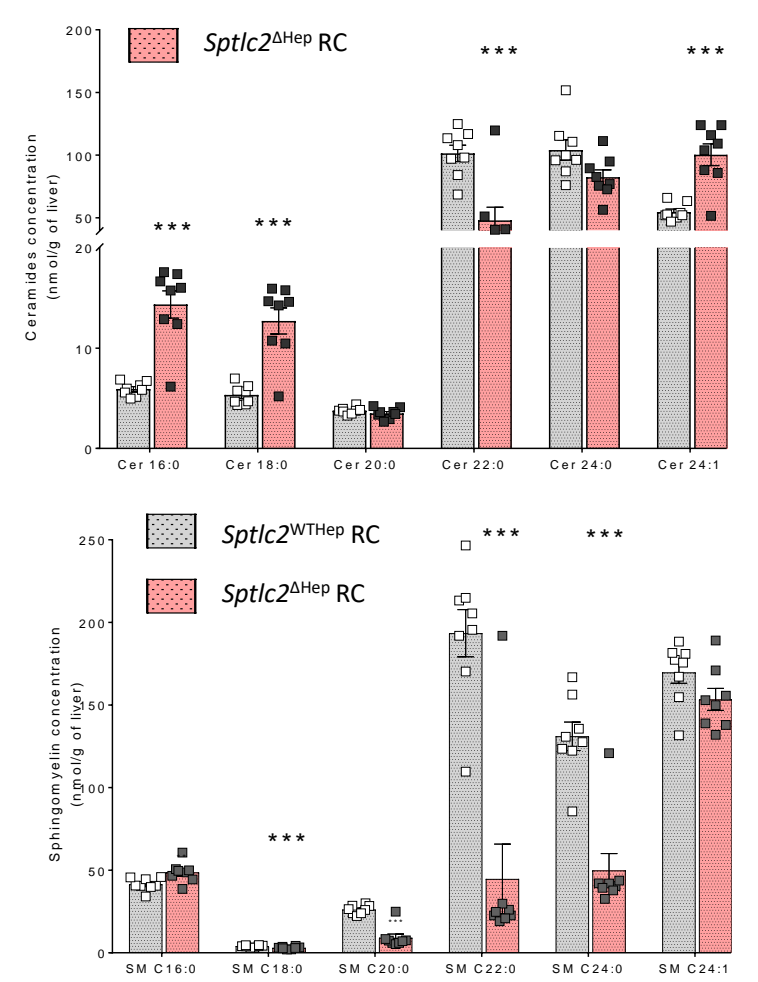

F.

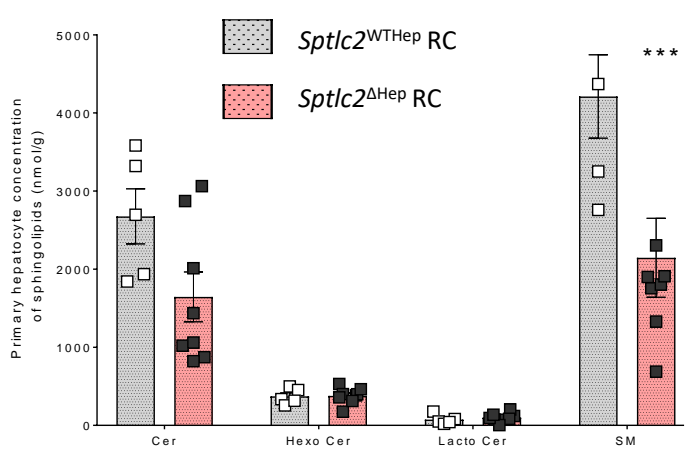

H.
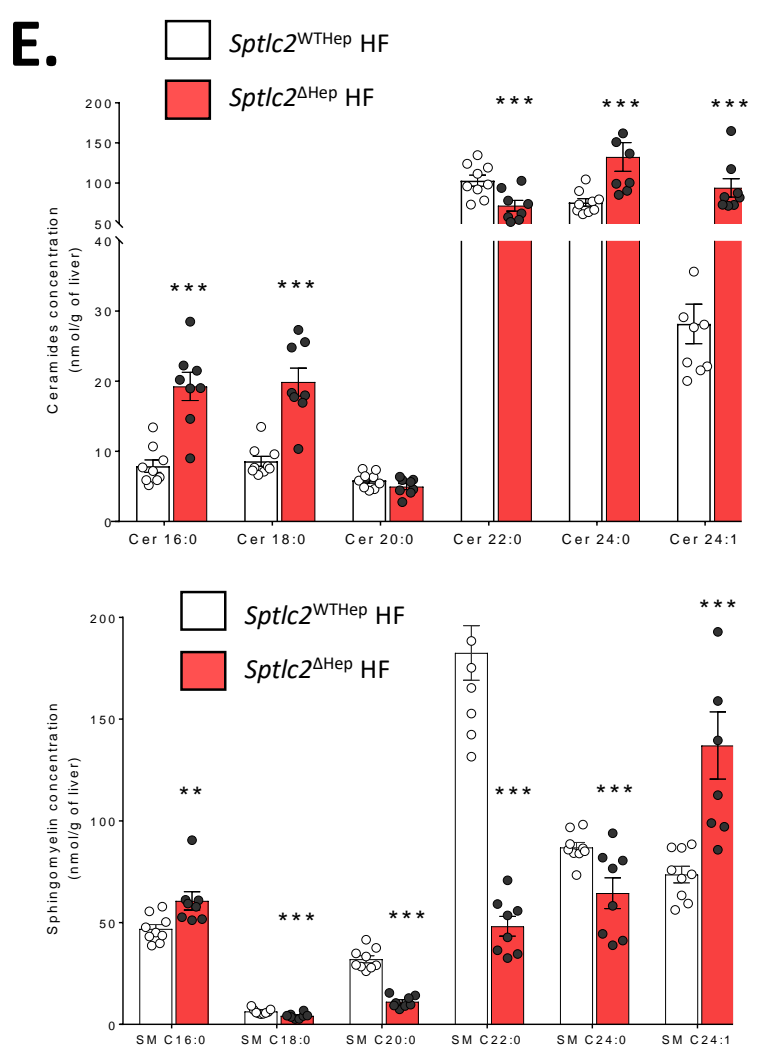

G.

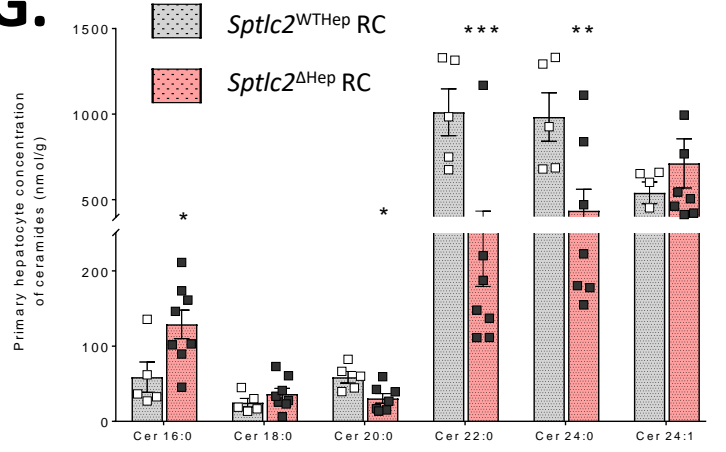




\section{Figure S2.}
A. $\quad$ spt/c2 ${ }^{\text {WTHee }} \mathrm{RC}$
B.
Sptlc2 ${ }^{\text {WTHep }} \mathrm{HF}$
Spt/c2 $2^{\text {मHер }} \mathrm{RC}$
Spt/c $2^{\Delta \mathrm{Hep}} \mathrm{HF}$

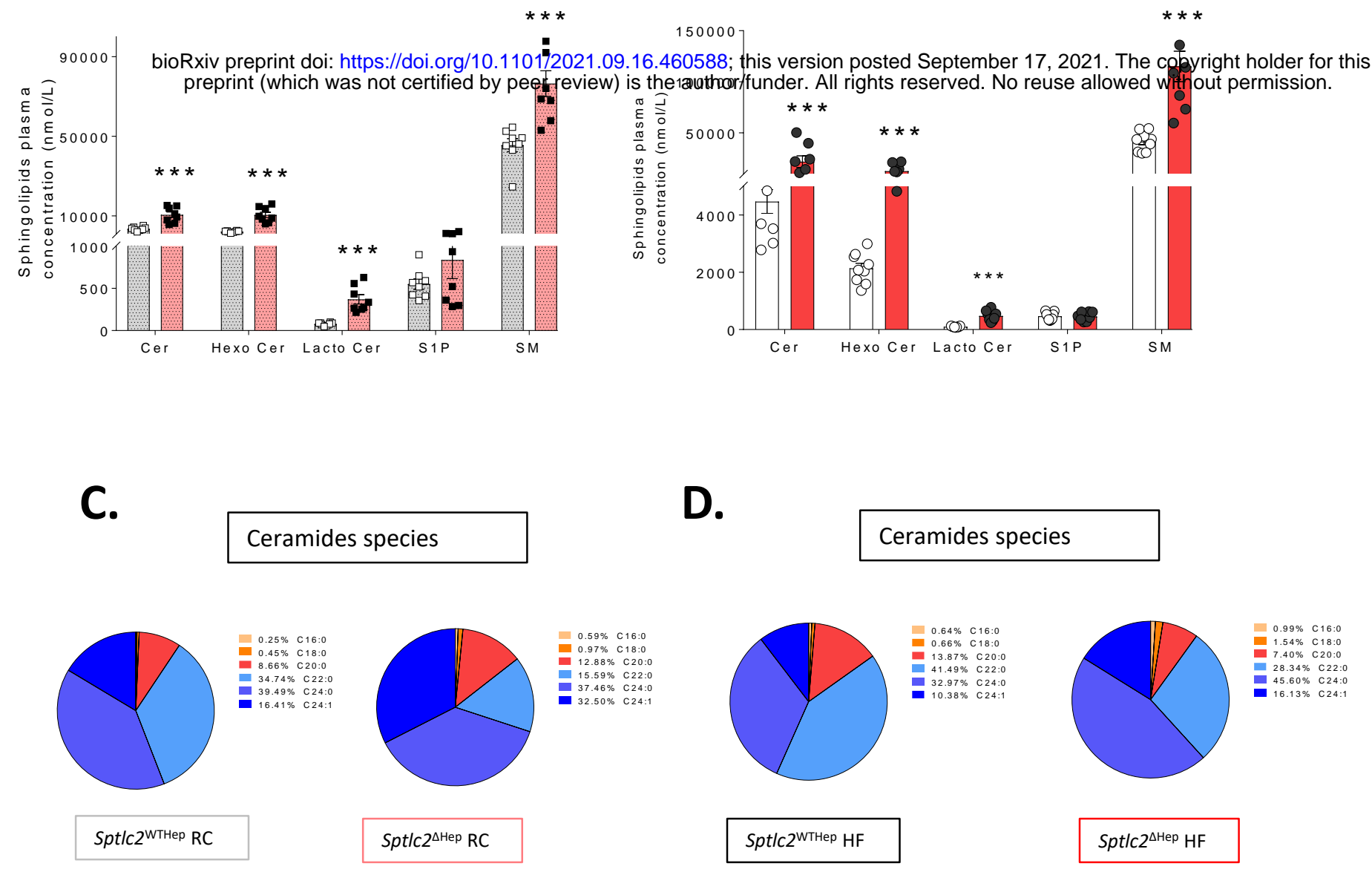




\section{Figure S3.}

A

B

SptIc2 WTHep RC

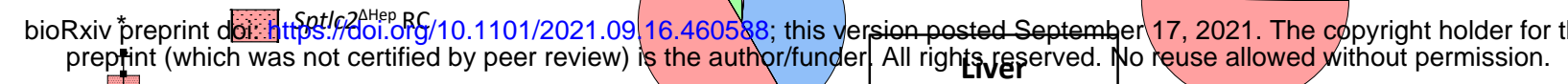

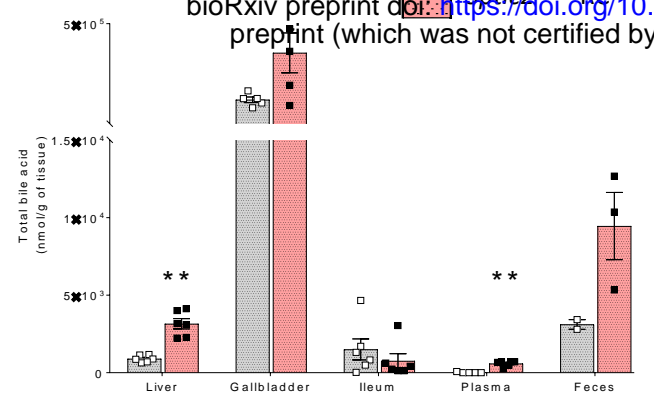

C

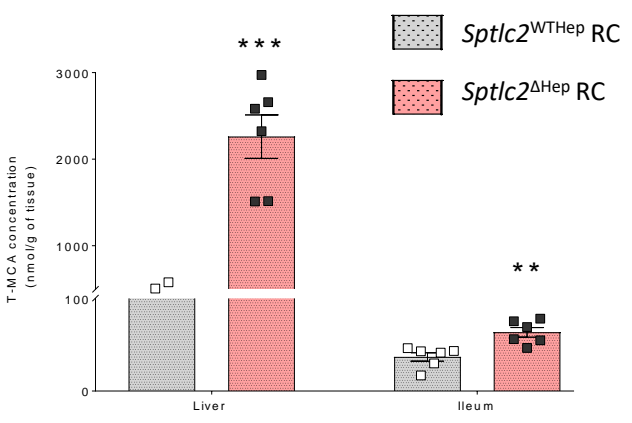

D

Sptlc2 ${ }^{\text {WTHep }} \mathrm{RC}$

Sptlc2 ${ }^{\text {LHep }} \mathrm{RC}$

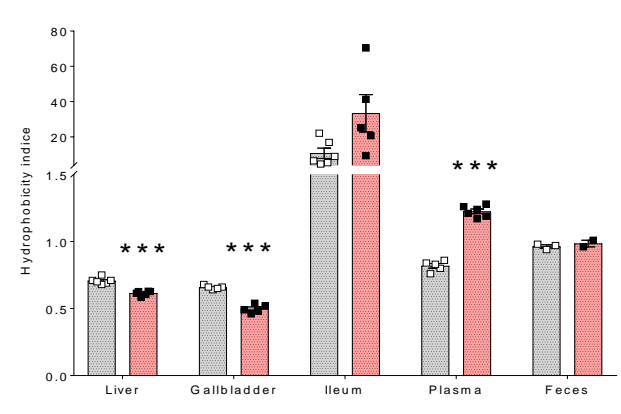

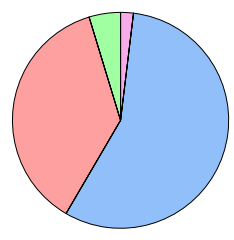

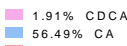

= $36.89 \% \mathrm{bMCA}$

Gallbalder

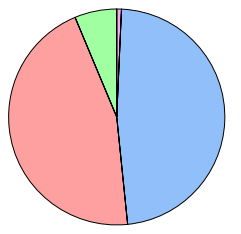

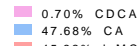

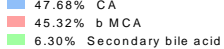

Ileum

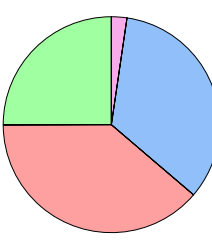

$2.31 \% 0$
$-20 D C A$

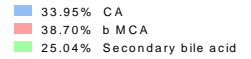

Plasma

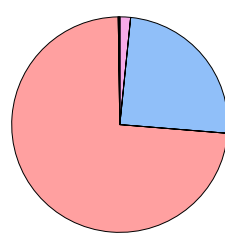

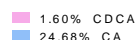

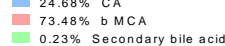

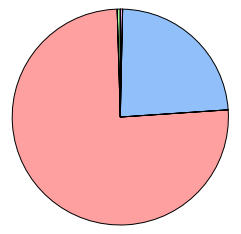

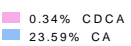

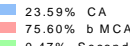

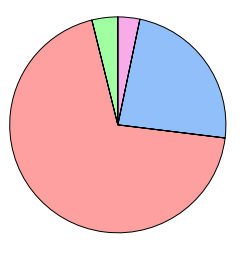

$3.28 \%$ CDCA

$23.67 \% \mathrm{CA}$
$69.19 \% \mathrm{~b}$
$-19 \mathrm{MC}$
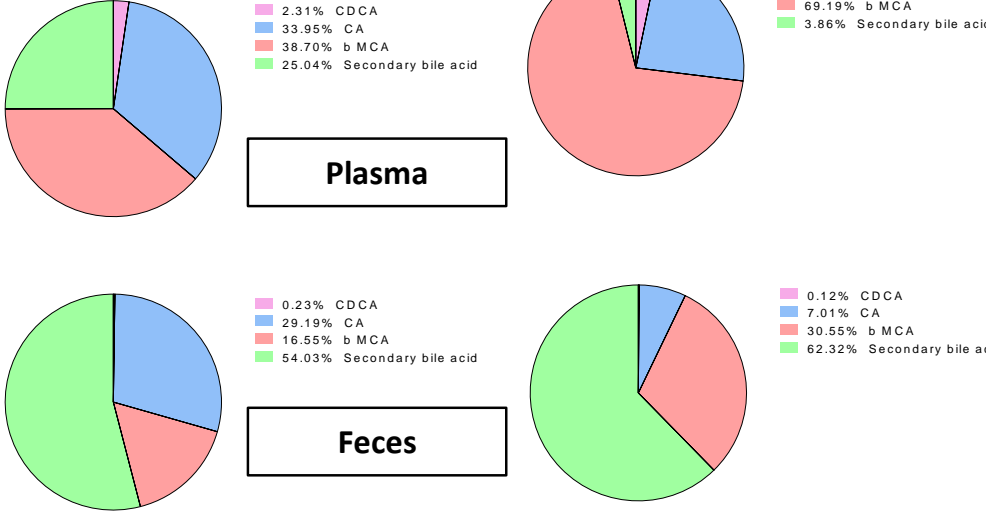

$0.23 \%$ CDCA
$29.19 \%$ CA
$29.5 \%$

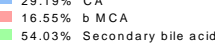

Feces

SptIc2 $^{\text {WTHep HF }}$

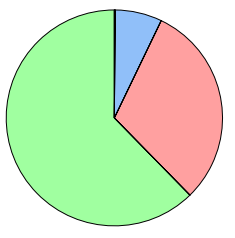

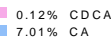

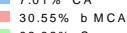




\section{Figure S4}
A.
Spt/c2 ${ }^{\text {WTHep }} \mathrm{RC}$
Spt/c2 $2^{\Delta \text { Hep } R C}$
B. $\square$ sptlc2WTHep HF

$24 \mathrm{~h}$ Fasted
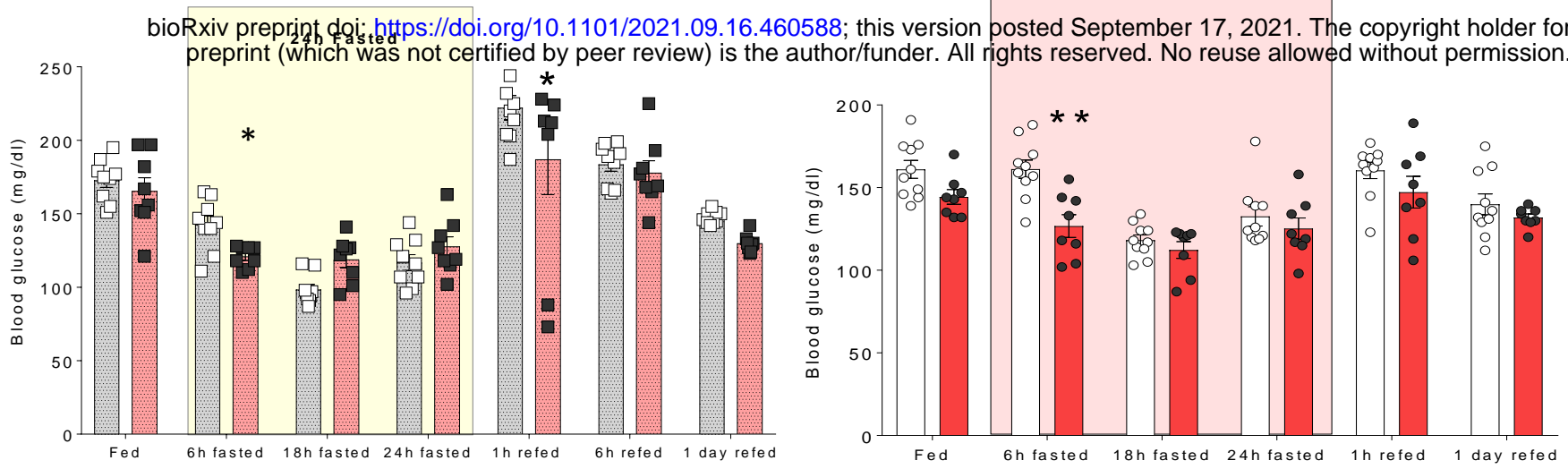

C.

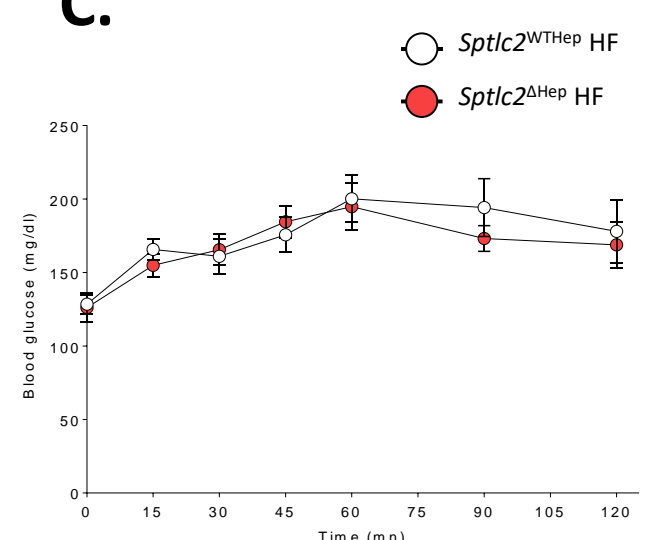

D.

$\square$ Spt/c2 ${ }^{\text {WTHep }} \mathrm{HF}$

Sptlc2 $2^{\Delta \text { Hep }} \mathrm{HF}$
E.

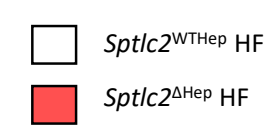

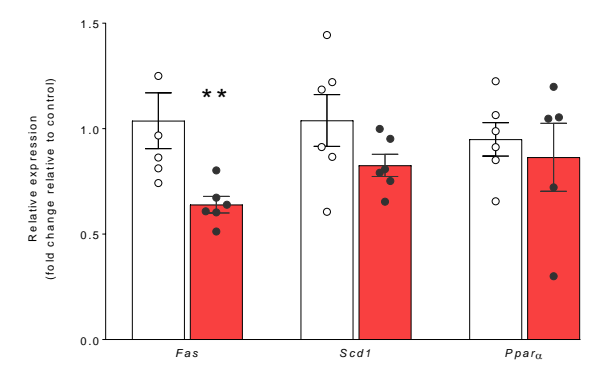

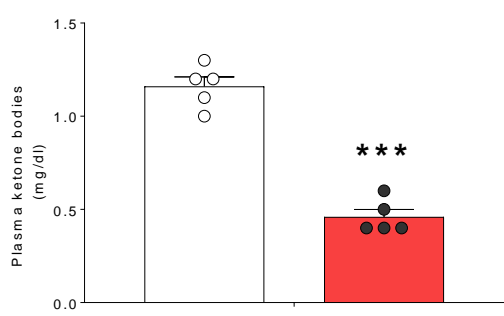




\section{Figure S5}
A.
Spt/c2 ${ }^{\text {WTHep }} \mathrm{RC}$
B.

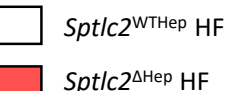
B. $\square$ sptlc2 $2^{\text {मHep }} \mathrm{HF}$

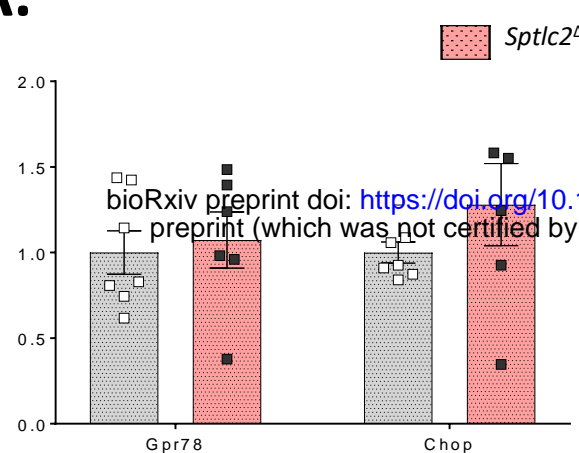

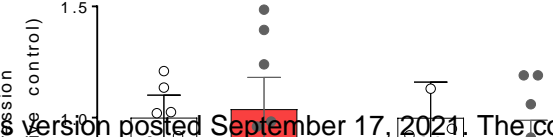

thiš Lersion posiled September 17, 2020, The copyright holder for this - preprint (which was not certified by peer review) is the author/fuâder. All rights reserved. No reuse allowed without permission.

Gpr78

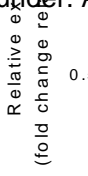

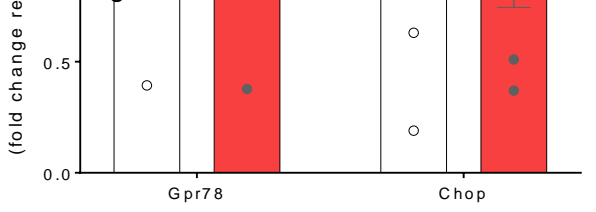

